\title{
The many formulae for the number of Latin rectangles
}

\author{
Douglas S. Stones* \\ School of Mathematical Sciences \\ Monash University \\ VIC 3800 Australia \\ the_empty_element@yahoo.com
}

Submitted: Apr 29, 2010; Accepted: Jun 1, 2010; Published: Jun 14, 2010

Mathematics Subject Classifications: 05B15, 00-02, 05A05, 01A05

\begin{abstract}
A $k \times n$ Latin rectangle $L$ is a $k \times n$ array, with symbols from a set of cardinality $n$, such that each row and each column contains only distinct symbols. If $k=n$ then $L$ is a Latin square. Let $L_{k, n}$ be the number of $k \times n$ Latin rectangles. We survey (a) the many combinatorial objects equivalent to Latin squares, (b) the known bounds on $L_{k, n}$ and approximations for $L_{n}$, (c) congruences satisfied by $L_{k, n}$ and (d) the many published formulae for $L_{k, n}$ and related numbers. We also describe in detail the method of Sade in finding $L_{7,7}$, an important milestone in the enumeration of Latin squares, but which was privately published in French. Doyle's formula for $L_{k, n}$ is given in a closed form and is used to compute previously unpublished values of $L_{4, n}$, $L_{5, n}$ and $L_{6, n}$. We reproduce the three formulae for $L_{k, n}$ by Fu that were published in Chinese. We give a formula for $L_{k, n}$ that contains, as special cases, formulae of (a) Fu, (b) Shao and Wei and (c) McKay and Wanless. We also introduce a new equation for $L_{k, n}$ whose complexity lies in computing subgraphs of the rook's graph.
\end{abstract}

\section{Introduction}

A $k \times n$ Latin rectangle is a $k \times n$ array $L$, with symbols from $\mathbb{Z}_{n}$, such that each row and each column contains only distinct symbols. If $k=n$ then $L$ is a Latin square of order $n$. Let $L_{k, n}$ be the number of $k \times n$ Latin rectangles. As we will see, the exact value of $L_{k, n}$ can be computed only for small values of $k$ or $n$.

The main aim of this paper is to provide a survey of the many formulae involving $L_{k, n}$. The structure of this paper is as follows. In the remainder of this section we summarise the enumeration of $L_{n, n}$ for small $n$. In Section 2 we identify several combinatorial objects that

\footnotetext{
* Supported by the Monash Faculty of Science Postgraduate Publications Award. Supported by ARC grant DP0662946.
} 
are equivalent to Latin squares or Latin rectangles. We also introduce some important equivalence relations amongst Latin squares and Latin rectangles. In Section 3 we survey the bounds for $L_{k, n}$ and compare the bounds for $L_{n, n}$ in a table for $5 \leqslant n \leqslant 20$. In Section 4 we discuss congruences satisfied by $L_{k, n}$. In Section 5 we list several explicit formulae for $L_{k, n}$ and $L_{n, n}$. We also use a formula of Doyle to find values of $L_{k, n}$ for $k \in\{4,5,6\}$. In Section 6 we give a detailed discussion of the method used by Sade in finding $L_{7,7}$ and describe the modern algorithm by McKay and Wanless that was used to find $L_{11,11}$. In Section 7 we survey the asymptotic formulae for $L_{k, n}$. We give some concluding remarks in Section 8.

In this paper, we assume $k \leqslant n$. We will index the rows of $L$ by $\{0,1, \ldots, k-1\} \subseteq \mathbb{Z}_{n}$, the columns of $L$ by $\mathbb{Z}_{n}$ and take the symbol set to be $\mathbb{Z}_{n}$. A Latin rectangle is called normalised if the first row is $(0,1, \ldots, n-1)$, and reduced if the first row is $(0,1, \ldots, n-1)$ and the first column is $(0,1, \ldots, k-1)^{T}$. Let $K_{k, n}$ denote the number of normalised $k \times n$ Latin rectangles and let $R_{k, n}$ denote the number of reduced $k \times n$ Latin rectangles. In the case of Latin squares, the numbers $L_{n, n}, K_{n, n}$ and $R_{n, n}$ will be denoted $L_{n}, K_{n}$ and $R_{n}$, respectively. The three numbers $L_{k, n}, K_{k, n}$ and $R_{k, n}$ are related by

$$
L_{k, n}=n ! K_{k, n}=\frac{n !(n-1) !}{(n-k) !} R_{k, n} .
$$

In particular

$$
L_{n}=n ! K_{n}=n !(n-1) ! R_{n} .
$$

Observe that (a) $K_{n}$ is also the number of Latin squares $L=\left(l_{i j}\right)$ of order $n$ with $l_{i i}=0$ for all $i \in \mathbb{Z}_{n}$ and (b) $R_{n}$ also is the number of normalised Latin squares $L=\left(l_{i j}\right)$ of order $n$ with $l_{i i}=0$ for all $i \in \mathbb{Z}_{n}$ [83, Thm 7.21].

The use of the term "reduced" goes back at least to MacMahon [87], and was adopted, for example, by Fisher and Yates [47], Dénes and Keedwell [29, 32] and Laywine and Mullen [83]. Euler [43] instead used the term quarrés réguliers or "regular square." Some authors use "normalised" [95, 176], "standardized" [41], "standard" or "in standard form" [114] in place of what we call "reduced." Similarly, our definition of "normalised" also has some alternative names; for example "standardised" [34], "in the standard form" [8], "semi-normalised" [176] and "reduced" [20, 26, 64, 121], which can be confusing. Some authors avoid this problem by not assigning names to reduced or normalised Latin squares, for example [21, 59, 124, 163].

The number of $k \times n$ normalised Latin rectangles $L=\left(l_{i j}\right)$ satisfying $l_{00}<l_{10}<\cdots<$ $l_{(k-1) 0}$ is the number of $k \times n$ Latin rectangles with the first row and column in order; it is given by $L_{k, n} /(n !(k-1) !)$. For $k<n$ this is not, in general, the number of reduced $k \times n$ Latin rectangles, as given by (1). In [162] this type of Latin rectangle was called "reduced." A notion of "very reduced" was considered by Moser [104], which was later generalised to " $i-j$ reduced" by Mullen [105] and Hamilton and Mullen [67].

Recently, McKay and Wanless [96] published a table of values for $R_{k, n}$ when $2 \leqslant k \leqslant$ $n \leqslant 11$, which was obtained by lengthy computer enumerations (this table is reproduced in Figure 3; we omit $R_{n-1, n}=R_{n}$ and $R_{1, n}=1$ ). Figure 1 reproduces the values of $R_{n}$ for $1 \leqslant n \leqslant 11$ and alongside is a list of relevant references. As can be seen in the 
table, much research has been put into the enumeration of $R_{n}$ over many years and some surveys of its history were provided by Dénes and Keedwell [29, Sec. 4.3], McKay and Wanless [96] and McKay, Meynert and Myrvold [94]. It is possible that Clausen found $R_{6}$ in 1842 (see [80] for a discussion). The value of $R_{12}$ is currently unknown, but the estimate $R_{12} \approx 1.62 \cdot 10^{44}$ was one of the estimates given by McKay and Rogoyski [95]. Zhang and $\mathrm{Ma}$ [178] and Kuznetsov [82] later gave estimates for $R_{n}$ for $n \leqslant 20$ which agree with the estimates in [95]. We tabulate these estimates in Figure 2.

\begin{tabular}{|r|rll|}
\hline$n$ & $R_{n}$ & Year & References \\
\hline 1 & 1 & \\
2 & 1 & \\
3 & 1 & & \\
4 & 1 & & \\
5 & 4 & & \\
6 & 56 & 1782 & {$[21,43,89]$} \\
7 & 9408 & 1890 & {$[47,48,70,132,134,158,174]$} \\
8 & 16942080 & 1948 & {$[48,55,114,125,127,133,173]$} \\
9 & 535281401856 & 1967 & {$[4,81,107,168]$} \\
10 & 377597570964258816 & 1975 & {$[7,107]$} \\
11 & 5363937773277371298119673540771840 & 2005 & {$[96]$} \\
\hline
\end{tabular}

Figure 1: $R_{n}$ for $1 \leqslant n \leqslant 11$.

\begin{tabular}{|r|r|r|rrr|}
\hline$n$ & $\begin{array}{r}\text { McKay, Rogoyski } \\
R_{n} \approx\end{array}$ & $\begin{array}{r}\text { Zhang, Ma } \\
R_{n} \approx\end{array}$ & $R_{n} \approx$ & $\begin{array}{c}\text { Kuznetsov } \\
\text { confidence interval }\end{array}$ & \%err. \\
\hline 12 & $1.62 \cdot 10^{44}$ & $1.622 \cdot 10^{44}$ & $1.612 \cdot 10^{44}$ & $\left(1.596 \cdot 10^{44}, 1.629 \cdot 10^{44}\right)$ & 1 \\
13 & $2.51 \cdot 10^{56}$ & $2.514 \cdot 10^{56}$ & $2.489 \cdot 10^{56}$ & $\left(2.465 \cdot 10^{56}, 2.515 \cdot 10^{56}\right)$ & 1 \\
14 & $2.33 \cdot 10^{70}$ & $2.332 \cdot 10^{70}$ & $2.323 \cdot 10^{70}$ & $\left(2.300 \cdot 10^{70}, 2.347 \cdot 10^{70}\right)$ & 1 \\
15 & $1.5 \cdot 10^{86}$ & $1.516 \cdot 10^{86}$ & $1.516 \cdot 10^{86}$ & $\left(1.499 \cdot 10^{86}, 1.531 \cdot 10^{86}\right)$ & 1 \\
16 & & $7.898 \cdot 10^{103}$ & $8.081 \cdot 10^{103}$ & $\left(7.920 \cdot 10^{103}, 8.242 \cdot 10^{103}\right)$ & 2 \\
17 & & $3.768 \cdot 10^{123}$ & $3.717 \cdot 10^{123}$ & $\left(3.642 \cdot 10^{123}, 3.791 \cdot 10^{123}\right)$ & 2 \\
18 & & $1.869 \cdot 10^{145}$ & $1.828 \cdot 10^{145}$ & $\left(1.773 \cdot 10^{145}, 1.883 \cdot 10^{145}\right)$ & 3 \\
19 & & $1.073 \cdot 10^{169}$ & $1.103 \cdot 10^{169}$ & $\left(1.059 \cdot 10^{169}, 1.147 \cdot 10^{169}\right)$ & 4 \\
20 & & $7.991 \cdot 10^{194}$ & $7.647 \cdot 10^{194}$ & $\left(7.264 \cdot 10^{194}, 8.028 \cdot 10^{194}\right)$ & 5 \\
50 & & $3.06 \cdot 10^{2123}$ & & & \\
100 & & $1.78 \cdot 10^{11396}$ & & & \\
\hline
\end{tabular}

Figure 2: Estimates for $R_{n}$. 


\begin{tabular}{|r|r|}
\hline$n, k$ & $R_{k, n}$ \\
\hline 3,2 & 1 \\
\hline 4,2 & 3 \\
3 & 4 \\
\hline 5,2 & 11 \\
3 & 46 \\
4 & 56 \\
\hline 6,2 & 53 \\
3 & 1064 \\
4 & 6552 \\
5 & 9408 \\
\hline 7,2 & 309 \\
3 & 35792 \\
4 & 1293216 \\
5 & 11270400 \\
6 & 16942080 \\
\hline 8,2 & 2119 \\
3 & 1673792 \\
4 & 420909504 \\
5 & 27206658048 \\
6 & 335390189568 \\
7 & 535281401856 \\
\hline
\end{tabular}

\begin{tabular}{|r|r|}
\hline$n, k$ & $R_{k, n}$ \\
\hline 9,2 & 16687 \\
3 & 103443808 \\
4 & 207624560256 \\
5 & 112681643083776 \\
6 & 12952605404381184 \\
7 & 224382967916691456 \\
8 & 377597570964258816 \\
\hline 10,2 & 148329 \\
3 & 8154999232 \\
4 & 147174521059584 \\
5 & 746988383076286464 \\
6 & 870735405591003709440 \\
7 & 177144296983054185922560 \\
8 & 4292039421591854273003520 \\
9 & 7580721483160132811489280 \\
\hline 11,2 & 1468457 \\
3 & 798030483328 \\
4 & 143968880078466048 \\
5 & 7533492323047902093312 \\
6 & 96299552373292505158778880 \\
7 & 240123216475173515502173552640 \\
8 & 86108204357787266780858343751680 \\
9 & 2905990310033882693113989027594240 \\
10 & 5363937773277371298119673540771840 \\
\hline
\end{tabular}

Figure 3: $R_{k, n}$ for $2 \leqslant k<n \leqslant 11$. 


\section{Equivalence}

In this section we will identify some combinatorial objects equivalent (in some sense) to Latin squares. Many of the objects listed in this section were identified by [6, 25, 29, 83]. Our primary focus will be in finding formulae for $L_{n}, K_{n}$ or $R_{n}$. We are not able to produce a complete list of the combinatorial objects equivalent to Latin squares, nor is it likely to be possible to produce such a list.

\subsection{Isotopism and parastrophy}

We are motivated by Bailey and Cameron [6] to accompany our discussion of objects equivalent to Latin squares with a discussion on the symmetry of the object.

Let $S_{n}$ be the symmetric group acting on $\mathbb{Z}_{n}$. For any Latin square $L=\left(l_{i j}\right)$ of order $n$ an ordered triplet of permutations $\theta=(\alpha, \beta, \gamma) \in S_{n} \times S_{n} \times S_{n}$ will denote a mapping of $L$ such that the rows of $L$ are permuted according to $\alpha$, the columns of $L$ are permuted according to $\beta$ and the symbols of $L$ are permuted according to $\gamma$. In other words, $\theta(L)=\left(l_{i j}^{\prime}\right)$ is the Latin square defined by

$$
l_{i j}^{\prime}=\gamma\left(l_{\alpha^{-1}(i) \beta^{-1}(j)}\right)
$$

for all $i, j \in \mathbb{Z}_{n}$. The mapping $\theta$ is called an isotopism. The group of all isotopisms is called the isotopism group. The identity permutation will be denoted $\varepsilon$. Any isotopism other than $(\varepsilon, \varepsilon, \varepsilon)$ is non-trivial.

Let $L$ and $L^{\prime}$ be Latin squares of order $n$. If there exists an isotopism $\theta$ such that $\theta(L)=L^{\prime}$, then $L$ and $L^{\prime}$ are said to be isotopic. The set of all Latin squares isotopic to $L$ is called the isotopy class of $L$. If $\theta(L)=L$, then $\theta$ is said to be an autotopism of $L$. The group of all autotopisms of $L$ is called the autotopism group of $L$, which we will denote $\operatorname{Atop}(L)$. If $\theta=(\alpha, \beta, \gamma)$ is an isotopism such that $\alpha=\beta=\gamma$, then $\theta$ is said to be an isomorphism. The group of all isomorphisms is called the isomorphism group. The set of all Latin squares isomorphic to $L$ is called the isomorphism class of $L$. If $\theta$ is an isomorphism and an autotopism of $L$ then $\theta$ is said to be an automorphism of $L$. The group of all automorphisms of $L$ is called the automorphism group of $L$, denoted $\operatorname{Aut}(L)$. Clearly, $\operatorname{Aut}(L)$ is a subgroup of $\operatorname{Atop}(L)$. Classifying which isotopisms are autotopisms of some Latin square is a largely open problem [44, 77, 78, 144, 153]. Given a Latin square $L=\left(l_{i j}\right)$ of order $n$ we can construct a set of $n^{2}$ ordered triplets

$$
O=\left\{\left(i, j, l_{i j}\right): i, j \in \mathbb{Z}_{n}\right\}
$$

called the orthogonal array of $L$. Conversely, any set $O$ of $n^{2} \operatorname{triplets}\left(i, j, l_{i j}\right) \in \mathbb{Z}_{n} \times \mathbb{Z}_{n} \times$ $\mathbb{Z}_{n}$, such that distinct triplets differ in at least two coordinates, gives rise to a Latin square $L=\left(l_{i j}\right)$. Any element of the orthogonal array $O$ of $L$ is called an entry of $L$. There are six, not necessarily distinct, Latin squares that can be constructed from $L$ by uniformly permuting the coordinates of each entry in $O$ and each is called a parastrophe of $L$. We use $\lambda \in\{\varepsilon,(r c),(r s),(c s),(r c s),(r s c)\}$ to permute the coordinates of each entry in $O$, where 
$r, c$ and $s$ correspond to the first, second and third coordinates, respectively. We use $L^{\lambda}$ to denote the parastrophe of $L$ induced by $\lambda$ and call $\{\varepsilon,(r c),(r s),(c s),(r c s),(r s c)\}$ under composition the parastrophy group. For example, $L^{(r c)}$ is the matrix transpose of $L$. For $k \times n$ Latin rectangles $L$ with $k<n$, we can similarly construct a set of $k n$ entries $O$ from $L$. However, it is only sensible to consider the (cs)-parastrophe of $L$.

Typically, "conjugate" [149, 150] is used in place of "parastrophe" [128, 139]. Here we use "parastrophe" to match the author's PhD thesis [153]. Group-theoretic conjugation plays an important role in the study of autotopisms [77, 153] and there are other notions of conjugacy in the study of quasigroups $[74,75]$ (quasigroups will be introduced in Section 2.2). Norton [114], for example, used the term "adjugate," but this terminology is rarely adopted in modern times. The term "adjugate" also has a use in linear algebra.

The term "parastrophe" is due to J. B. Shaw [139], but the concept goes back to E. Schröder [136] and even L. Euler [43]. Parastrophes have been considered by E. Schröder [136, 137] and Schönhardt [134] under the name "Koordiniert", by R. H. Bruck $[13,14]$ as the "associated" algebras, by I. M. H. Etherington [42] as "transposes", by H. A. Thurston [159] as "equasigroups" and by D. A. Norton and S. K. Stein [112, 113, 149, 150] as "conjugates".

- Sade (translated) [128]

The one name that I very much regret we did not advocate strongly enough in connection with enumeration is "parastrophe" (see Sade [128]) rather than "conjugate" (invented by Stein $[149,150]$ ).

- Keedwell (private communication 2010)

The main class (or species) of $L$ is the set of all Latin squares that are isotopic to some parastrophe of $L$. If $L$ and $L^{\prime}$ are within the same main class, then they are said to be paratopic. A map that combines both isotopism and parastrophy is called a paratopism. The group of all paratopisms is called the paratopism group. If $\tau$ is a paratopism such that $\tau(L)=L$ then $\tau$ is said to be an autoparatopism of $L$. The group of all autoparatopisms of $L$ is called the autoparatopism group of $L$, which we will denote $\operatorname{Apar}(L)$.

The term "species" [7, 55, 114, 119, 127, 168] was popular until circa 1974 when Dénes and Keedwell published their book [29], which instead used the now popular term "main class" [25, 32, 69, 81, 94, 96]. It appears Dénes and Keedwell derived this term from Schönhardt [134], who used the German "Grundklasse." McKay, Meynert and Myrvold [94] also noted the synonym "paratopy class," but this is rarely used.

Several other subgroups of the paratopism group are of importance. For instance, McKay, Meynert and Myrvold [94] considered the type of $L$, which is the set of all Latin squares that are either isotopic to $L$ or isotopic to $L^{(r c)}$. We will call the group combining isotopism with $(r c)$-parastrophy the type group. Another example are isotopisms of the form $\theta=(\alpha, \beta, \varepsilon)$, which are called principal isotopisms. Principal isotopisms have been studied, for example, by Ganfornina [50]. 
We depict some of the subgroup structure of the paratopism group in Figure 4, with arrows denoting subgroups. The first row of groups vary only with $n$, the second row of groups varies with the Latin square $L$ (which is of order $n$ ) and the third row of groups are independent of both $L$ and $n$.

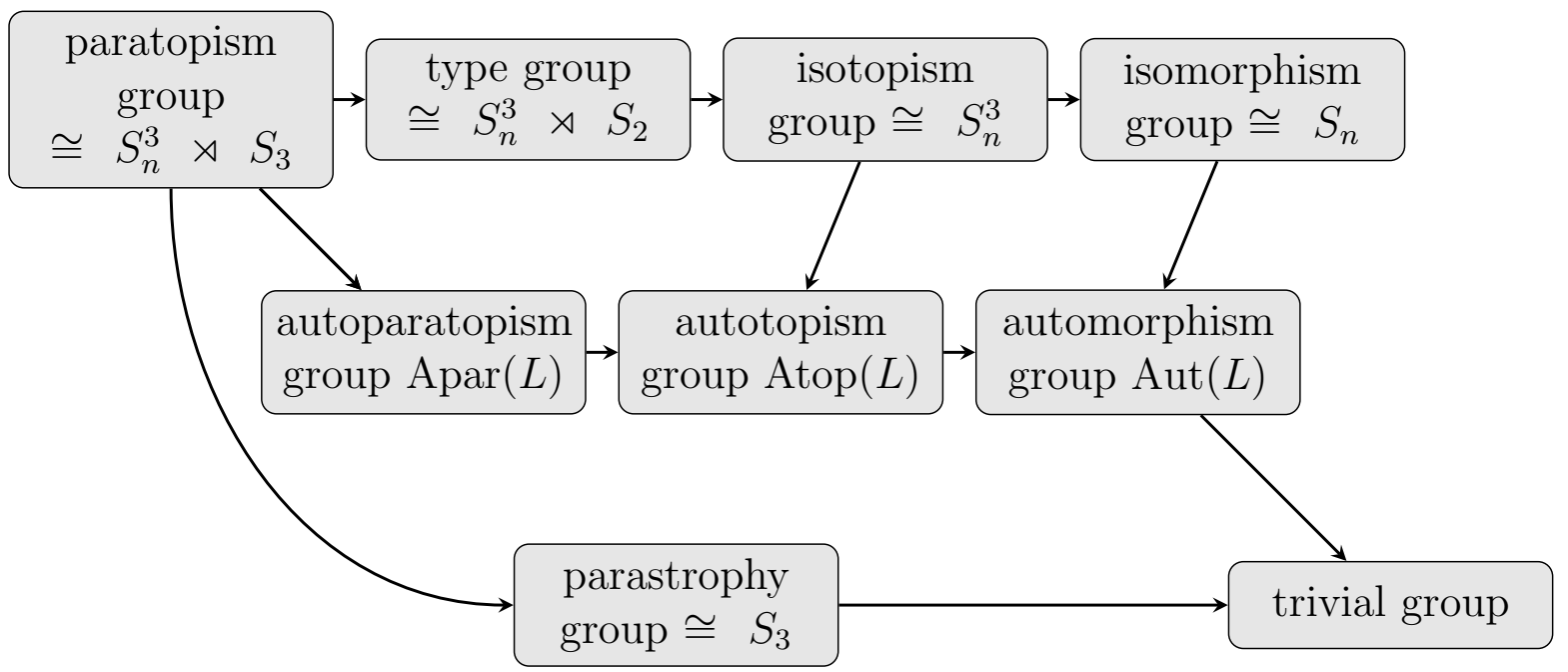

Figure 4: Some important subgroups of the paratopism group, where $L$ is a Latin square of order $n$.

McKay, Meynert and Myrvold [94] gave a construction from $L$ of three graphs with automorphism groups that are isomorphic to $\operatorname{Aut}(L), \operatorname{Atop}(L)$ and $\operatorname{Apar}(L)$. The LOOPS [108, 109] package for GAP [51] implements a completely different algorithm for finding the automorphism group of a Latin square. For orders $1 \leqslant n \leqslant 10$, the number of (a) main classes, (b) types, (c) isotopy classes, (d) isomorphism classes and (e) isomorphism classes containing a reduced Latin square was also given by [94] along with enumeration formulae and a survey of the "sorry history of the subject". Hulpke, Kaski and Östergård [69] reported these numbers for order 11. These numbers are reproduced in Figures 5 and 7 along with a list of relevant references. None of these numbers alone provide sufficient information to find $L_{n}$.

Various notation has been used instead of $\operatorname{Atop}(L)$ and $\operatorname{Apar}(L)$. We list some examples in Figure 6. Since there is no consensus on notation, the decision to use $\operatorname{Atop}(L)$ and $\operatorname{Apar}(L)$ in this paper is based on the author's personal preference.

\subsection{Quasigroups}

A quasigroup $(Q, \oplus)$ of order $n$ is a set $Q$ of cardinality $n$ together with a binary operation $\oplus$, such that for all $g, h \in Q$, the equations $x \oplus g=h$ and $g \oplus y=h$ have unique solutions with $x, y \in Q$. If $(Q, \oplus)$ possesses an identity element $e$, that is $e$ satisfies $e \oplus g=g=g \oplus e$ for all $g \in Q$, then $Q$ is called a loop. 


\begin{tabular}{|r|rrr|}
\hline$n$ & Main classes & Types & Isotopy classes \\
\hline 1 & 1 & 1 & 1 \\
2 & 1 & 1 & 1 \\
3 & 1 & 1 & 1 \\
4 & 2 & 2 & 2 \\
5 & 2 & 2 & 2 \\
6 & 12 & 17 & 22 \\
7 & 147 & 324 & 564 \\
8 & 283657 & 842227 & 1676267 \\
9 & 19270853541 & 57810418543 & 115618721533 \\
10 & 34817397894749939 & 104452188344901572 & 208904371354363006 \\
11 & 2036029552582883134196099 & 6108088657705958932053657 & 12216177315369229261482540 \\
\hline & References: $[4,11,12,47,69,81,94,114,119,125,132,134,168]$ & \\
\hline
\end{tabular}

Figure 5: The number of main classes, types and isotopy classes of Latin squares of order $n$.

\begin{tabular}{|rr|lcc|}
\hline & & $\operatorname{Aut}(L)$ & $\operatorname{Atop}(L)$ & $\operatorname{Apar}(L)$ \\
\hline 2007 & Hämäläinen and Cavenagh [66] & & $\operatorname{Aut}(L)$ & \\
2007 & Csörgö, Drápal and Kinyon [27] & $\operatorname{Aut}(L)$ & $\operatorname{Atp}(L)$ & \\
c. 2006 & Falcón et al. [44, 45, 50] & & $\mathcal{U}(L)$ & \\
c. 2005 & McKay et al. [94, 96] & $\operatorname{Aut}(L)$ & $\operatorname{Is}(L)$ & $\operatorname{Par}(L)$ \\
2004 & Kinyon, Kunen and Phillips [79] & & $\operatorname{Atop}(L)$ & \\
1990 & Kolesova, Lam and Thiel [81] & & $G_{L}$ & $G_{L}$ \\
c. 1967 & Sade [129, 130, 131] & & $\mathfrak{A}(L)$ & \\
\hline
\end{tabular}

Figure 6: Table of synonyms for $\operatorname{Aut}(L), \operatorname{Atop}(L)$ and $\operatorname{Apar}(L)$.

If $(Q, \oplus)$ is a quasigroup and $\triangleleft$ is a total order on $Q$, then we call $(Q, \oplus, \triangleleft)$ an ordered quasigroup. The Cayley table of an ordered quasigroup $(Q, \oplus, \triangleleft)$ is the matrix $L=\left(l_{i j}\right)$ such that $l_{i j}=i \oplus j$, where the rows and columns of $L$ are indexed by $Q$ in the order defined by $\triangleleft$. Hence $L_{n}$ is the number of ordered quasigroups on a set $Q$ of cardinality $n$ with total order $\triangleleft$. If $(Q, \oplus, \triangleleft)$ is an ordered loop such that the identity $e$ is the minimum under $\triangleleft$, then its Cayley table is a reduced Latin square. Hence $R_{n}$ is the number of ordered loops on a set $Q$ of cardinality $n$ with identity $e \in Q$ and total order $\triangleleft$ with minimum $e$.

For any permutation $\alpha$ of $Q$, we may define a quasigroup $(Q, \star)$ by $\alpha(i) \star \alpha(j)=\alpha(i \oplus j)$ for all $i, j \in Q$. We say that $(Q, \star)$ is isomorphic to $(Q, \oplus)$ and call the set of quasigroups isomorphic to $(Q, \oplus)$ the isomorphism class of $(Q, \oplus)$. Let $\triangleleft$ be any total order on $Q$. Let $L$ and $L^{\prime}$ be the unique Cayley tables of the ordered quasigroups $(Q, \oplus, \triangleleft)$ and $(Q, \star, \triangleleft)$, respectively. Then $\theta(L)=L^{\prime}$ where $\theta=(\alpha, \alpha, \alpha)$ by (3), that is, $L$ is isomorphic to $L^{\prime}$. It follows that an isomorphism class of Latin squares is precisely the set of Cayley tables of an isomorphism class of quasigroups with a fixed total order $\triangleleft$. In fact, the definition of 
isomorphism amongst Latin squares stems from isomorphism amongst quasigroups.

The number of isomorphism classes of quasigroups is the number of isomorphism classes of Latin squares of order $n$. Curiously, the number of isomorphism classes of quasigroups is odd for all $1 \leqslant n \leqslant 17$ except when $n=12$ [151]. A result of [96] implies that the number of isomorphism classes of quasigroups is asymptotic to $L_{n} / n !=K_{n}$. In each isomorphism class of quasigroups there (a) might not be a loop, (b) might be one loop or (c) might be more than one loop. The number of isomorphism classes of Latin squares that contain a reduced Latin square is the number of isomorphism classes of loops (since every quasigroup isomorphic to a loop is also a loop). These numbers are listed for $n \leqslant 11$ in Figure 7, sourced from [69, 94], along with a list of relevant references.

\begin{tabular}{|r|rr|}
\hline$n$ & Loops & Quasigroups \\
\hline 1 & 1 & 1 \\
2 & 1 & 1 \\
3 & 1 & 5 \\
4 & 2 & 35 \\
5 & 6 & 1411 \\
6 & 109 & 1130531 \\
7 & 23746 & 12198455835 \\
8 & 106228849 & 2697818331680661 \\
9 & 9365022303540 & 15224734061438247321497 \\
10 & 20890436195945769617 & 2750892211809150446995735533513 \\
11 & 14781574551580444452849321016 & 19464657391668924966791023043937578299025 \\
\hline & References: $[1,9,17,29,69,94,131,134]$, Bower, Guérin and "QSCGZ" [94] \\
\hline
\end{tabular}

Figure 7: The number of isomorphism classes of loops of order $n$ and the number of isomorphism classes of quasigroups of order $n$, for $1 \leqslant n \leqslant 11$.

In Figure 8 we reproduce the list, given by Bailey and Cameron [6], of isomorphism class representatives of Latin squares of order 3. These Latin squares are not isomorphic, but they are isotopic. In fact, there is only one isotopy class of Latin squares of order 3.

$$
\left(\begin{array}{lll}
0 & 2 & 1 \\
2 & 1 & 0 \\
1 & 0 & 2
\end{array}\right),\left(\begin{array}{lll}
0 & 1 & 2 \\
1 & 2 & 0 \\
2 & 0 & 1
\end{array}\right),\left(\begin{array}{lll}
0 & 1 & 2 \\
2 & 0 & 1 \\
1 & 2 & 0
\end{array}\right),\left(\begin{array}{lll}
0 & 2 & 1 \\
1 & 0 & 2 \\
2 & 1 & 0
\end{array}\right),\left(\begin{array}{lll}
1 & 0 & 2 \\
0 & 2 & 1 \\
2 & 1 & 0
\end{array}\right)
$$

Figure 8: A Latin square from each isomorphism class of order 3.

\subsection{Graphs}

In this section we identify some graph-theoretic objects that are equivalent to Latin squares (see also [29, Sec. 9.1] and [83, Ch. 7]). 


\subsubsection{Rook's graphs.}

Let $G=G_{k, n}$ be the graph with vertex set $\{(i, j): 0 \leqslant i \leqslant k-1$ and $0 \leqslant j \leqslant n-1\}$ and edges between distinct $(i, j)$ and $\left(i^{\prime}, j^{\prime}\right)$ whenever $i=i^{\prime}$ or $j=j^{\prime}$. We will call $G$ a rook's graph since edges represent legal moves by a rook on a $k \times n$ chess board. There are other names for $G$, for example $G$ is (a) the line graph of the complete bipartite graph (with parts of cardinality $k$ and $n$ ) and (b) the graph Cartesian product of the complete graphs on $k$ and $n$ vertices. As usual we assume $k \leqslant n$.

A $k \times n$ Latin rectangle $L=\left(l_{i j}\right)$ corresponds to a proper vertex-colouring of $G$ with colour set $\mathbb{Z}_{n}$, with vertex $(i, j)$ receiving colour $l_{i j}$. This observation was made by Athreya, Pranesachar and Singhi [5]. For example, Figure 9 is $G_{3,4}$ with an example of a proper vertex-colouring from the colour set $\mathbb{Z}_{4}$. Hence $L_{k, n}$ is the number of proper vertex-colourings of $G$ with colour set $\mathbb{Z}_{n}$. Equivalently, $L_{k, n}$ is the chromatic polynomial $P(G, x)$ evaluated at $x=n$, that is, $L_{k, n}=P\left(G_{k, n}, n\right)$.

The number of $k \times n$ matrices with at most $x$ distinct symbols in total and without repeated symbols in each row and column is enumerated by $P(G, x)$. The enumeration of this type of generalised Latin rectangle was also considered by Light Jr. [86] and Nechvatal [111]. In Figure 10 we list $P(G, x)$ for some small values of $k$ and $n$ that were computed by Kerri Morgan (private communication).

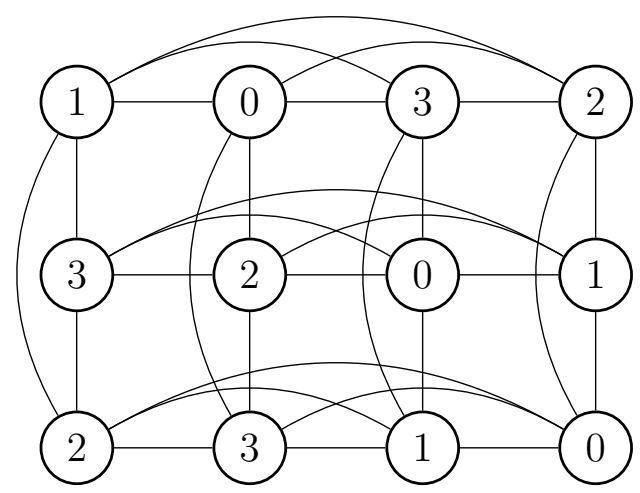

Figure 9: The graph $G_{3,4}$ with a proper vertex-colouring from the colour set $\mathbb{Z}_{4}$.

\subsubsection{Latin square graphs.}

Let $L=\left(l_{i j}\right)$ be a Latin square of order $n$. Let $H=H(L)$ be the graph with vertex set $\left\{(i, j): i, j \in \mathbb{Z}_{n}\right\}$ and an edge between distinct $(i, j)$ and $\left(i^{\prime}, j^{\prime}\right)$ whenever $i=i^{\prime}$ or $j=j^{\prime}$ or $l_{i j}=l_{i^{\prime} j^{\prime}}$. The graph $H$ is called a Latin square graph of order $n$.

We say a graph $G$ is $(v, a, b, c)$-strongly regular if (a) $G$ has $v$ vertices, (b) every vertex has $a$ neighbours, (c) every pair of adjacent vertices has $b$ common neighbours and (d) every pair of non-adjacent vertices has $c$ common neighbours. A Latin square graph is $\left(n^{2}, 3(n-1), n, 6\right)$-strongly regular. The following theorem was attributed to Bruck [16] (see also [15]) by Bailey and Cameron [6, Pro. 3]. 


\begin{tabular}{|rr|r|}
\hline$k$ & $n$ & $P\left(G_{k, n}, x\right)$ \\
\hline 2 & 2 & $x(x-1)\left(x^{2}-3 x+3\right)$ \\
2 & 3 & $x(x-1)(x-2)(x-3)\left(x^{4}-10 x^{3}+41 x^{2}-84 x+73\right)$ \\
2 & 4 & $x(x-1)(x-2)(x-3)(x-4)\left(x^{5}-15 x^{4}+95 x^{3}-325 x^{2}+609 x-501\right)$ \\
2 & 5 & $x(x-1)(x-2)\left(x^{6}-15 x^{5}+100 x^{4}-381 x^{3}+877 x^{2}-1152 x+688\right)$ \\
\hline 3 & 3 & $x(x-1)(x-2)(x-3)\left(x^{8}-24 x^{7}+264 x^{6}-1746 x^{5}+7620 x^{4}-22512 x^{3}\right.$ \\
3 & 4 & $\left.+43939 x^{2}-51630 x+27808\right)$ \\
& & \\
3 & 5 & $x(x-1)(x-2)(x-3)(x-4)\left(x^{10}-35 x^{9}+570 x^{8}-5710 x^{7}+39098 x^{6}-191728 x^{5}\right.$ \\
& & $\left.+683055 x^{4}-1746375 x^{3}+3063456 x^{2}-3321652 x+1684912\right)$ \\
\hline 4 & 4 & $x(x-1)(x-2)(x-3)\left(x^{12}-42 x^{11}+833 x^{10}-10338 x^{9}+89589 x^{8}-572046 x^{7}+2762671 x^{6}\right.$ \\
& & $\left.-10172046 x^{5}+28328427 x^{4}-58124022 x^{3}+83236871 x^{2}-74505978 x+31430160\right)$ \\
\hline
\end{tabular}

Figure 10: $P\left(G_{k, n}, x\right)$ for some small values of $k$ and $n$.

Theorem 2.1. If $n \geqslant 24$ then any $\left(n^{2}, 3(n-1), n, 6\right)$-strongly regular graph is a Latin square graph. Furthermore, if (a) $L$ is a Latin square of order $n \geqslant 5$, (b) $H$ is the Latin square graph of $L$ and (c) $H^{\prime}$ is a graph isomorphic to $H$, then $H^{\prime}$ is the Latin square graph of a Latin square $L^{\prime}$ paratopic to $L$.

It follows that, for $n \geqslant 24$, the number of isomorphism classes of $\left(n^{2}, 3(n-1), n, 6\right)$ strongly regular graphs is the number of main classes of Latin squares of order $n$. The automorphisms of Latin square graphs were studied by Phelps $[116,117]$.

\subsubsection{Proper edge-colourings of the complete bipartite graph.}

Let $G$ be the balanced complete bipartite graph with vertex bipartition $\left\{u_{0}, u_{1}, \ldots, u_{n-1}\right\} \cup$ $\left\{w_{0}, w_{1}, \ldots, w_{n-1}\right\}$. Let $C$ be a proper edge-colouring of $G$ with edge colour set $\mathbb{Z}_{n}$. The edges of colour $s$ define a permutation of $\mathbb{Z}_{n}$ by $i \mapsto j$ whenever $u_{i}$ is adjacent to $w_{j}$ by an edge of colour $s$. So we can construct a Latin square $L=L(C)=\left(l_{i j}\right)$ from $C$ with $l_{i j}=s$ whenever $u_{i}$ is adjacent to $w_{j}$ by an edge of colour $s$. Hence $L_{n}$ is the number of proper edge-colourings of $G$ with edge colour set $\mathbb{Z}_{n}$.

The group $\operatorname{Aut}(G) \times S_{n}$ acts on the set of proper edge-colourings of $G$; with $(\tau, \gamma) \in$ Aut $(G) \times S_{n}$ permuting the vertices of $G$ according to $\tau$ and the edge colours according to $\gamma$. In fact, $\operatorname{Aut}(G) \times S_{n}$ is isomorphic to the type group (see Section 2.1). Let $C$ be an arbitrary proper edge-colouring of $G$. The orbit of $C$ under $\operatorname{Aut}(G) \times S_{n}$ corresponds to the type of $L(C)$. Therefore the number of non-isomorphic edge-colourings of $G$ is the number of types of Latin squares of order $n$.

A one-factor of a graph (in this case $G$ ) is a 1-regular spanning subgraph. A decomposition of $G$ is a set of subgraphs of $G$ whose edge sets partition the edge set of $G$. In particular, a one-factorisation of $G$ is a decomposition of $G$ into a set of one-factors. Given a one-factorisation of $G$, we can construct $n$ ! proper edge-colourings by assigning a distinct colour of $\mathbb{Z}_{n}$ to each one-factor and then colouring each edge in $G$ according to the colour of one-factor to which it belongs. Consequently, $K_{n}=L_{n} / n$ ! is the number 
of one-factorisations of $G$. The number of non-isomorphic one-factorisations of $G$ is the number of types of Latin squares of order $n$.

Figure 11 depicts a one-factorisation of the balanced complete bipartite graph $G$ on $2 n=10$ vertices. The first column of vertices is $u_{0}, u_{1}, \ldots, u_{n-1}$ and the second is $w_{0}, w_{1}, \ldots, w_{n-1}$, with both in descending order. To illustrate the correspondence with Latin squares, the vertices $u_{i}$ are marked $j$ whenever $u_{i}$ is adjacent to $w_{j}$. Note that Figure 11 identifies the Latin square defined by $l_{i s}=j$, that is, $L(C)^{(c s)}$.

Dénes and Keedwell [30, 31] and Laywine and Mullen [83] discussed one-factorisations of the complete bipartite graph (see also [29] and [32]). Wanless et al. [18, 19, 91, 164, $166,167]$ studied the Latin squares formed from certain one-factorisations of $G$.

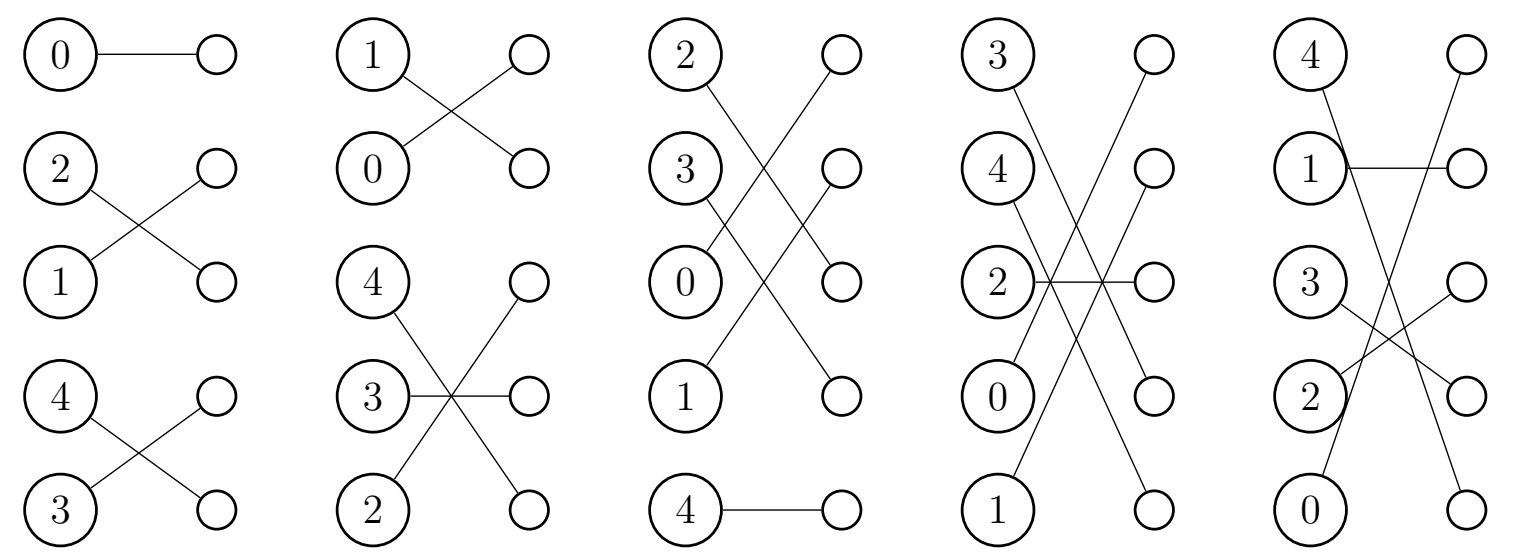

Figure 11: A one-factorisation of a complete bipartite graph.

\subsubsection{One-factorisations of the complete directed graph.}

A set $S$ of permutations of $\mathbb{Z}_{n}$ is called sharply transitive if for all $i, s \in \mathbb{Z}_{n}$ there is a unique $\sigma \in S$ such that $\sigma(i)=s$. It follows that $|S|=n$. We define $\sigma_{j} \in S$ to be the permutation that maps 0 to $j$. We can construct a normalised Latin square $L=\left(l_{i j}\right)$ of order $n$ from $S$ by assigning $l_{i j}=\sigma_{j}(i)$. Moreover, if $\varepsilon \in S$ then $L$ is a reduced Latin square. Hence $K_{n}$ is the number of sharply transitive sets of $\mathbb{Z}_{n}$ and $R_{n}$ is the number of sharply transitive sets $S$ of $\mathbb{Z}_{n}$ with $\varepsilon \in S$.

A one-factorisation of a directed graph $G$ is a decomposition of $G$ into subgraphs in which every vertex has in-degree and out-degree 1.

Let $G$ be the loop-free complete directed graph on the vertex set $\mathbb{Z}_{n}$. Assume that $\varepsilon \in S$. Each non-trivial $\sigma \in S$ is equivalent to the subgraph of $G$ with an edge from each $i \in \mathbb{Z}_{n}$ to $\sigma(i)$. Together, the non-trivial $\sigma \in S$ yield a one-factorisation of $G$. Conversely, given a one-factorisation of $G$ we may reverse this process to construct a sharply transitive set of permutations $S=\left\{\sigma_{j}\right\}_{j \in \mathbb{Z}_{n}}$ with $\sigma_{0}=\varepsilon$. Hence $R_{n}$ is the number of one-factorisations of $G$. This equivalence was noticed in [83, pp. 112-113].

Let $G^{\prime}$ be the complete directed graph on $n$ vertices, with a single loop on each vertex. A one-factorisation of $G^{\prime}$ corresponds to a sharply transitive set $S=\left\{\sigma_{j}\right\}_{j \in \mathbb{Z}_{n}}$, 
but this time we do not necessarily have $\sigma_{0}=\varepsilon$. Consequently, $K_{n}$ is the number of onefactorisations of $G^{\prime}$. This equivalence was also noticed in [83, pp. 111-112]. In Figure 12 we give an example of a one-factorisation of $G^{\prime}$ on 3 vertices; if there is an edge from vertex $i$ to vertex $j$, then we mark vertex $i$ with $j$ to highlight the correspondence with Latin squares.

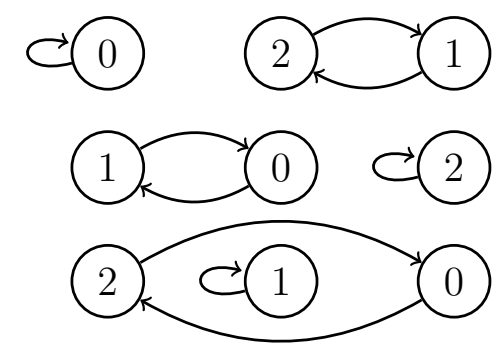

Figure 12: A one-factorisation of $G^{\prime}$ on 3 vertices.

\subsubsection{Triangle decompositions of the complete tripartite graph.}

Let $G$ be the balanced complete tripartite graph with vertex partition $R \cup C \cup S$ with $|R|=|C|=|S|=n$. We will consider a triangle of $G$ to be any triplet in $R \times C \times S$. The orthogonal array of $L$ therefore defines a decomposition of $G$ into triangles. Hence $L_{n}$ is the number of decompositions of $G$ into triangles. Figure 13 gives an example of a triangulation of the balanced complete tripartite graph on 6 vertices; identically labelled vertices are identified.

Colbourn [24] used the NP-completeness of the problem of decomposing a tripartite graph into triangles to show that the problem of partial Latin square completion is also NP-complete.

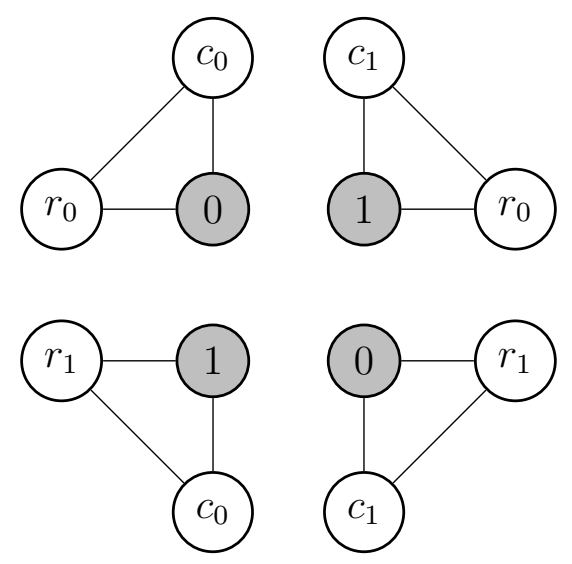

Figure 13: A triangulation of a complete tripartite graph on 6 vertices. 


\subsection{Miscellany}

\subsubsection{3-nets and transversal designs.}

A 3-net $[6,29,68,72]$ is an incidence structure with $n^{2}$ points and $3 n$ lines such that (a) each line contains $n$ points and each point lies on 3 lines, (b) each pair of points lie on at most one line and (c) the lines can be partitioned into 3 families of $n$ lines, each of which is a partition of the set of points, with each pair of lines from distinct families intersecting at a unique point. A Latin square $L$ forms a 3-net with its orthogonal array as the set of points and lines corresponding to the rows, columns and symbols of $L$. Condition (c) implies that $L$ can be recovered from the 3-net [6].

A transversal design is the dual of a 3 -net. It has $3 n$ points and $n^{2}$ lines such that (a) each line contains 3 points and each point lies on $n$ lines, (b) each pair of points lie on at most one line and (c) the points can be partitioned into 3 families of $n$ points, with each pair of points from different families lying on a unique line and each line containing one point from each family.

Isomorphism amongst 3-nets and transversal designs corresponds to paratopism of Latin squares. Therefore, the number of non-isomorphic 3-nets is the number of nonisomorphic transversal designs, and is also the number of main classes of Latin squares.

\subsubsection{Error-detecting codes.}

We can write the orthogonal array of a Latin square $L=\left(l_{i j}\right)$ as an $n^{2} \times 3$ array with each row equal to $\left(i, j, l_{i j}\right)$ for some $i, j \in \mathbb{Z}_{n}$. It has the property that any pair of distinct rows differs by at least two entries. Such an array is called a 1-error-detecting code [29, p. 354]. The rows are referred to as codewords, the symbol set is called the alphabet and the word length is 3, the number of columns. It is straightforward to construct an orthogonal array of a Latin square from a 1-error-detecting code with these parameters. Hence $L_{n}$ is the number of 1-error-detecting codes with $n^{2}$ codewords of word length 3 and alphabet of size $n$.

\subsubsection{Permutation cubes.}

Let $L$ be a Latin square. Then $L$ corresponds to the $n \times n \times n(0,1)$-array $M=\left(m_{i j k}\right)$ with $m_{i j k}=1$ whenever $l_{i j}=k$. Equivalently $M$ indicates the position of $n^{2}$ mutually non-attacking rooks on an $n \times n \times n$ chess board. Hence $L_{n}$ is the number of such arrays $M$ and the number of arrangements of $n^{2}$ mutually non-attacking rooks on an $n \times n \times n$ chess board.

\section{Bounds}

In this section we discuss the known bounds for $R_{n}$. We will see that the best known bounds for $R_{n}$ are still quite poor. We can easily find a super-exponential lower bound on $R_{n}$. In fact, for any $k \geqslant 2, R_{k, n}$ increases super-exponentially as $n \rightarrow \infty$. To show this, 
observe that $R_{k, n} \geqslant R_{k^{\prime}, n}$ whenever $k^{\prime} \leqslant k \leqslant n$. A derangement is a permutation without fixed points. When $n \geqslant k$ we have $R_{k, n} \geqslant R_{2, n}=D_{n} /(n-1)$, where $D_{n}$ is the number of derangements on a set of cardinality $n$. It is well-known that $D_{n} \sim \exp (-1) \cdot n$ !. Hence $R_{k, n}$ increases super-exponentially with $n$ and $R_{n} \geqslant R_{k, n}$ when $n \geqslant k$.

To study the bounds on $R_{k, n}$, we will need to introduce the permanent function for square matrices. The permanent of a square matrix, $M=\left(m_{i j}\right)_{n \times n}$ is defined as

$$
\operatorname{PER}(M)=\sum_{\sigma \in S_{n}} \prod_{i \in \mathbb{Z}_{n}} m_{i \sigma(i)}
$$

where $S_{n}$ is the symmetric group on $\mathbb{Z}_{n}$. The primary source of information for permanents is Minc [100, 101, 102], which was updated in [23]; see also his biography by Marcus [92].

Given a $k \times n$ Latin rectangle $L$ we can construct an $n \times n(0,1)$-matrix $T=\left(t_{i j}\right)$ such that $t_{i j}=1$ if and only if symbol $j$ does not occur in column $i$ in $L$. The matrix $T$ is called the template of $L$. We will index the rows and columns of $T$ by $\mathbb{Z}_{n}$. For any $\sigma \in S_{n}$, if $t_{i \sigma(i)}=1$ for all $i \in \mathbb{Z}_{n}$ then $L$ can be extended to a $(k+1) \times n$ Latin rectangle with the new row containing symbol $\sigma(i)$ in column $i$ for each $i \in \mathbb{Z}_{n}$. Therefore, the number of ways $L$ can be extended to a $(k+1) \times n$ Latin rectangle is $\operatorname{PER}(T)$.

Let $\Lambda_{n}^{s}$ denote the set of $(0,1)$-matrices with exactly $s$ non-zero entries in each row and column. It follows that

$$
\prod_{s=0}^{k-1} \min _{M \in \Lambda_{n}^{n-s}} \operatorname{PER}(M) \leqslant L_{k, n} \leqslant \prod_{s=0}^{k-1} \max _{M \in \Lambda_{n}^{n-s}} \operatorname{PER}(M) .
$$

Let $M=\left(m_{i j}\right)$ be a $(0,1)$-matrix and define the row sum $r_{i}=\sum_{j \in \mathbb{Z}_{n}} m_{i j}$ for all rows $i$. Hall Jr. [65] showed that if $\operatorname{PER}(M)>0$ then $\operatorname{PER}(M) \geqslant \min _{i \in \mathbb{Z}_{n}} r_{i}$ !. Jurkat and Ryser $[73,(12.33)]$ showed that $\operatorname{PER}(M) \geqslant \prod_{i=1}^{n} \max \left(0, r_{i}-i+1\right)$. Minc [99] showed that a result of Sinkhorn [140] implies that if $M \in \Lambda_{n}^{s}$ then $\operatorname{PER}(M) \geqslant n(s-3) / 3$ and improved this lower bound to $\operatorname{PER}(M) \geqslant n(s-2)+2$.

Minc [97] showed that $\operatorname{PER}(M) \leqslant \prod_{i \in \mathbb{Z}_{n}}\left(r_{i}+1\right) / 2$ with equality if and only if $M \in \Lambda_{n}^{1}$ which was subsequently improved $[98]$ to $\operatorname{PER}(M) \leqslant \prod_{i \in \mathbb{Z}_{n}}\left(r_{i}+\sqrt{2}\right) /(1+\sqrt{2})$. Brègman [10] (see also [135]) proved a conjecture of Minc [97] that $\operatorname{PER}(M) \leqslant \prod_{i \in \mathbb{Z}_{n}} r_{i} !^{1 / r_{i}}$. Liang and Bai [84] gave $\operatorname{PER}(M) \leqslant \prod_{i=0}^{n-1} \sqrt{a_{i}\left(r_{i}-a_{i}+1\right)}$ where $a_{i}=\min \left(\left\lceil\left(r_{i}+1\right) / 2\right\rceil,\lceil i / 2\rceil\right)$. A lower bound for the maximum permanent in $\Lambda_{n}^{s}$ was given by Wanless [165].

We can combine (4) with the above bounds on the permanent of matrices in $\Lambda_{n}^{s}$ to find bounds for $L_{k, n}$ and consequently $R_{k, n}$ by (1). We will now discuss some other bounds on $R_{n}$. Hall Jr. [65] gave the lower bound $R_{k, n} \geqslant \prod_{i=n-k+1}^{n-2} i$ !, which was also proved by Ryser [124, pp. 52-53]. Alter [3] gave the "crude upper bound" $R_{n} \leqslant(n-1) ! \prod_{i=1}^{n-2} i^{n-i-1} \cdot i$ ! An upper bound was also given by Duan [38], but it is no better than that of Alter for $n \geqslant 13$, although it appears Duan did not have access to Alter's paper. Smetaniuk [143] showed that $L_{n+1} \geqslant(n+1) ! L_{n}$ and therefore $R_{n+1} \geqslant(n-1) ! R_{n}$ by $(1)$. Van Lint and Wilson [163, Thm 17.2] showed that the van der Waerden Conjecture [161] (proved by $[39,46])$ implies that $L_{n} \geqslant n !^{2 n} n^{-n^{2}}$. Skau [141] showed that

$$
n !(1-k / n)^{n} L_{k, n} \leqslant L_{k+1, n} \leqslant(n-k) !^{n /(n-r)} L_{k, n} .
$$


A comparison of the discussed bounds for $R_{n}$ is given in Figure 14. We also include the known values of $R_{n}$ and the approximations by $[82,95,178]$ (see also Figure 2). The data in Section 5 is used to find Skau's bounds. It is clear there remains a large difference between the best upper and lower bounds on $R_{n}$. Judging from Figure 14, it appears that the best known upper and lower bounds on $R_{n}$ both have at least an exponential difference from $R_{n}$.

\begin{tabular}{|l|rrrrrrrrrrrrrrrr|}
\hline$n$ & 5 & 6 & 7 & 8 & 9 & 10 & 11 & 12 & 13 & 14 & 15 & 16 & 17 & 18 & 19 & 20 \\
\hline Lower bound by: & & & & & & & & & & & & & & & & \\
Hall Jr. & 2 & 3 & 5 & 8 & 12 & 16 & 22 & 28 & 36 & 45 & 54 & 65 & 77 & 91 & 105 & 121 \\
Smetaniuk & & & & & & & & 42 & 51 & 61 & 72 & 84 & 97 & 112 & 127 & 145 \\
Van Lint and Wilson & 1 & 2 & 4 & 8 & 13 & 20 & 28 & 37 & 48 & 61 & 76 & 93 & 112 & 132 & 155 & 180 \\
Skau & & & & & & & & 38 & 49 & 62 & 76 & 93 & 112 & 133 & 155 & 180 \\
\hline$R_{n}$ & 2 & 4 & 8 & 12 & 18 & 25 & 34 & & & & & & & & & \\
Approximation & 2 & 4 & 8 & 12 & 18 & 25 & 34 & 45 & 57 & 71 & 87 & 104 & 124 & 146 & 170 & 195 \\
\hline Upper bound by: & & & & & & & & & & & & & & & & \\
Skau & & & & & & & & 49 & 62 & 76 & 94 & 112 & 133 & 156 & 181 & 208 \\
Brègman, Minc & 3 & 5 & 9 & 14 & 21 & 29 & 38 & 49 & 63 & 77 & 94 & 113 & 134 & 156 & 181 & 208 \\
Liang and Bai & 2 & 5 & 9 & 14 & 20 & 29 & 38 & 50 & 63 & 79 & 96 & 116 & 137 & 161 & 187 & 215 \\
Alter & 4 & 7 & 12 & 19 & 27 & 37 & 50 & 64 & 80 & 99 & 119 & 142 & 168 & 196 & 226 & 259 \\
Duan & 2 & 5 & 10 & 16 & 25 & 35 & 48 & 63 & 81 & 101 & 123 & 149 & 177 & 208 & 242 & 278 \\
\hline
\end{tabular}

Figure 14: The number of decimal digits of some bounds on $R_{n}$, approximations of $R_{n}$ and the value of $R_{n}$ itself.

\section{Congruences}

The author's PhD thesis [153] was primarily concerned with congruences satisfied by $R_{k, n}$. The study of the number-theoretic properties of $R_{k, n}$ is partly motivated by Alter [3]. He asked the following three interesting questions concerning the divisibility of $R_{n}$ : (a) Do increasing powers of 2 divide $R_{n}$ ? (b) What is the highest power of 2 that will divide $R_{n}$ ? (c) Does 3 divide $R_{n}$ for all $n \geqslant 6$ ? The first and third of Alter's questions were answered by McKay and Wanless [96] with the following result.

Theorem 4.1. Let $m=\lfloor n / 2\rfloor$. For all $n \in \mathbb{N}, R_{n}$ is divisible by $m$ !. If $n$ is odd and $m+1$ is composite then $(m+1)$ ! divides $R_{n}$.

Theorem 4.1 shows that for any $d$, the largest $a$ such that $d^{a}$ divides $R_{n}$ increases at least linearly as $n \rightarrow \infty$. Subsequently, [157] gave the following theorem.

Theorem 4.2. Suppose $p$ is a prime and $n \geqslant 1$. If $d \geqslant k>p$ then $p^{\lfloor n / p\rfloor}$ divides $R_{k, n+d}$ and $K_{k, n}$. 
Theorem 4.2 shows that for any fixed $k$ and prime $p<k$, the largest $a$ such that $p^{a}$ divides $R_{k, n}$ increases at least linearly as $n \rightarrow \infty$. Theorem 4.1 is improved in some cases by the following theorem in [156].

Theorem 4.3. Suppose $n \geqslant 1, p$ is a prime and $c \geqslant 1$ such that $n / 2>(c-1) p$. Then $\operatorname{gcd}\left((n-c p-1) !^{2} R_{n-c p}, p^{c}\right)$ divides $R_{n}$.

In [157] it was also shown that

$$
R_{k, n} \equiv\left((-1)^{k-1}(k-1) !\right)^{n-1} \quad(\bmod n) .
$$

In particular this implies that $R_{n} \equiv 1(\bmod n)$ if $n$ is prime and $R_{n} \equiv 0(\bmod n)$ if $n$ is composite. In [154] and [155] it was shown that $R_{n+1} \equiv-2(\bmod n)$ if $n$ is an odd prime and $R_{n+1} \equiv 0(\bmod n)$ if $n$ is composite.

Riordan [121] gave the congruence $R_{3, n+p} \equiv 2 R_{3, n}(\bmod p)$ for all odd primes $p$, which was generalised by Carlitz [20] to $R_{3, n+t} \equiv 2^{t} R_{3, n}(\bmod t)$ for all $t \geqslant 1$. These recurrence congruences were generalised in [157] by the following theorem.

Theorem 4.4. If $k \leqslant n$ then $R_{k, n+t} \equiv\left((-1)^{k-1}(k-1) !\right)^{t} R_{k, n}(\bmod t)$ for all $t \geqslant 1$.

Theorem 4.4, in some cases, shows that $R_{k, n}$ is indivisible by some $t$ for all $n \geqslant k$, when $k$ is fixed and $t \geqslant k$ [157]. For example, the primes $p<100$ that do not divide $R_{3, n}$ for any $n \geqslant 3$ are $p \in\{3,5,11,29,37,41,43,53,67,79,83,97\}$ (see [153]).

A partial orthomorphism of $\mathbb{Z}_{n}$ is an injection $\sigma: S \rightarrow \mathbb{Z}_{n}$ such that $S \subseteq \mathbb{Z}_{n}$ and $\sigma(i)-i \not \equiv \sigma(j)-j(\bmod n)$ for distinct $i, j \in S$. We say $\sigma$ has deficit $d$ if $|S|=n-d$. Let $\chi(n, d)$ be the number of partial orthomorphisms $\sigma$ of $\mathbb{Z}_{n}$ of deficit $d$ such that $\sigma(i) \notin\{0, i\}$ for all $i \in S$. In [155] it is shown that, for prime $p$,

$$
R_{k, n} \equiv \chi(p, n-p) \frac{(n-p) !(n-p-1) !^{2}}{(n-k) !} R_{k-p, n-p} \quad(\bmod p) .
$$

In particular, for Latin squares $R_{p+d} \equiv d !(d-1) !^{2} \chi(p, d) R_{d}(\bmod p)$ for prime $p$ and all $d \geqslant 1$. The enumeration of partial orthomorphisms of $\mathbb{Z}_{n}$ was also described in [155], the results of which, when combined with Theorems 4.1 and 4.3 (using the Chinese Remainder Theorem), allow us to obtain the following congruences, for example.

$$
\begin{array}{ll}
R_{12} \equiv 50400(\bmod 55440), & R_{15} \equiv 8830080(\bmod 17297280), \\
R_{13} \equiv 342720(\bmod 720720), & R_{16} \equiv 7136640(\bmod 17297280), \\
R_{14} \equiv 428400(\bmod 720720), & R_{17} \equiv 95437440(\bmod 882161280) .
\end{array}
$$

It was also found that 5 divides $R_{7}$. Norton [114] gave an incomplete enumeration of the Latin squares of order 7 , having found 16927968 reduced Latin squares of order 7 (the total number is $16942080[127,126]$ which we will discuss in detail in Section 6.1.3). Since 5 does not divide 16927968 , we can deduce that $R_{7} \neq 16927968$ without finding the Latin squares that Norton missed. 
It is the purpose of this paper to present an extensive - possibly an exhaustive

- study of $7 \times 7$ Latin and higher squares.

- Norton [114]

Here, higher squares refers not to Latin squares of order greater than 7, but to GraecoLatin squares [29, Ch. 5], so Norton indeed acknowledged the possibility that his enumeration was incomplete.

Additionally, in [155] it was shown that, if $p$ is a prime, then

$$
\begin{aligned}
& R_{2 p-1} \equiv-R_{p-1}(\bmod p) \text { if } p \geqslant 2, \\
& R_{2 p-2} \equiv R_{p-2}(\bmod p) \text { if } p \geqslant 3, \\
& R_{2 p-3} \equiv-5 R_{p-3} / 13(\bmod p) \text { if } p \geqslant 5, \\
& R_{2 p-4} \equiv 29 R_{p-4} / 288(\bmod p) \text { if } p \geqslant 5, \\
& R_{2 p-5} \equiv-47 R_{p-5} / 2880(\bmod p) \text { if } p \geqslant 7, \\
& R_{2 p-6} \equiv 37 R_{p-6} / 19200(\bmod p) \text { if } p \geqslant 7 .
\end{aligned}
$$

In [153] it was shown that, for all primes $p, p^{a}$ divides $R_{n}$ where $a=n /(q-1)-$ $O\left(\log ^{2} n\right)$. Formulae involving the number of so-called even and odd Latin squares were discussed in $[36,37,56,156]$.

\section{General formulae}

In this section we will survey the general formulae for $L_{k, n}$ and $L_{n}$. For small $n$ the values of $K_{2, n}, K_{3, n}$ and $R_{4, n}$ are given by Sloane's [142] A000166, A000186 and A000573, respectively. Exact formulae for $L_{k, n}$ for $k \leqslant 4$ have been discussed in [157]. For example

$$
D_{n}=n ! \sum_{i=0}^{n} \frac{(-1)^{i}}{i !}=K_{2, n}=(n-1) R_{2, n}
$$

where $D_{n}$ is the number of derangements of $\mathbb{Z}_{n}$ (see [21] for example) and

$$
R_{3, n}=\sum_{i+j+k=n} n(n-3) !(-1)^{j} \frac{2^{k} i !}{k !}\left(\begin{array}{c}
3 i+j+2 \\
j
\end{array}\right)
$$

which was attributed to Yamamoto [121].

We now begin our survey of explicit formulae for $L_{k, n}$ for general $k$. First, we identify $L_{k, n}$ as a coefficient in a polynomial in $k n$ variables. Let $X=\left(x_{i j}\right)$ be a $k \times n$ matrix whose symbols are the $k n$ variables $x_{i j}$. We index the rows of $X$ by $[k]:=\{1,2, \ldots, k\}$ 
and the columns of $X$ by $[n]:=\{1,2, \ldots, n\}$, so $[k] \subseteq[n]$. Let $\mathcal{S}_{k, n}$ be the set of injections $\sigma:[k] \rightarrow[n]$. We define the permanent of the rectangular matrix $X$ to be

$$
\operatorname{PER}(X)=\sum_{\sigma \in \mathcal{S}_{k, n}} \prod_{i=1}^{k} x_{i \sigma(i)} .
$$

When $k=n$ this matches our definition of permanent for square matrices introduced in Section 3, except with different indices on $X$.

Theorem 5.1. $L_{k, n}$ is the coefficient of $\prod_{i=1}^{k} \prod_{j=1}^{n} x_{i j}$ in $\operatorname{PER}(X)^{n}$.

This theorem was noticed over a century ago by MacMahon [87] in the theory of symmetric functions encoded with $x_{i j}=\left(\alpha_{i}\right)^{2^{j-1}}$. He gives a different, but related formula in [89, Vol. 2, pp. 323-326] (also see his collected works [90]). We can obtain the value of $L_{k, n}$ from $\operatorname{PER}(X)^{n}$ by differentiation, for example

$$
L_{k, n}=\left.\left.\frac{\partial}{\partial x_{11}}\right|_{x_{11}=0} \cdots \frac{\partial}{\partial x_{k n}}\right|_{x_{k n}=0} \operatorname{PER}(X)^{n}
$$

which, when $k=n$, was one of Fu's [49] equations. MacMahon also used differentiation to "obliterate" the unwanted terms from $\operatorname{PER}(X)^{n}$ but in a different, more complicated, way to (6). The merit of MacMahon's formula has inspired much discussion.

The calculation will, no doubt, be laborious but that is here not to the point, as an enumeration problem may be considered to be solved when definite algebraical processes are set forth which lead to the solution.

- MacMahon [87]

I brought forward a new instrument of research in Combinatorial Analysis, and applied it to the complete solution of the great problem of the "Latin Square," which had proved a stumbling block to mathematicians since the time of Euler.

- MacMahon [88]

The problem of enumerating $n$ by $k$ Latin rectangles was solved formally by MacMahon using his operational methods.

- Erdős and Kaplansky [40]

A complete algebraic solution has been given by MacMahon in two forms, both of which involve the action of differential operators on an extended operand. If MacMahon's algebraic apparatus be actually put into operation, it will be found that different terms are written down, corresponding to all the different ways in which each row of the square could conceivably be filled up, that 
those arrangements which conflict with the conditions of the Latin square are ultimately obliterated, and those which conform to these conditions survive the final operation and each contribute unity to the result. The manipulation of the algebraic expressions, therefore, is considerably more laborious than the direct enumeration of the possible squares by a systematic and exhaustive series of trials.

- Fisher and Yates [47]

The use of MacMahon's result by mere mortals seems doomed.

- Riordan [122]

One should not wish to actually perform, even in a computer algebra package like Maple, MacMahon's counting method.

- Van Leijenhorst [162]

MacMahon's formula was nonetheless employed in a simplified form by Saxena [132, 133] to find $L_{6}$ and $L_{7}$, although these numbers were found earlier; see Figure 1 . Another proof of MacMahon's formula for $L_{n}$ was given by van Leijenhorst [162], who described it as both "beautiful" and "handsome." MacMahon had a particularly unorthodox life, even for a mathematician, which can be discovered in his biography [52].

Another way of extracting the value of $L_{k, n}$ from $\operatorname{PER}(X)^{n}$ was given by $\mathrm{Fu}$ [49], Shao and Wei [138] and McKay and Wanless [96]. We will write their formulae in a more general form in (8).

Let $\mathcal{B}_{k, n}$ be the set of $k \times n(0,1)$-matrices. As identified by $\mathrm{Fu}$ [49] and Shao and Wei [138], we can use Inclusion-Exclusion to obtain

$$
L_{k, n}=\sum_{A \in \mathcal{B}_{k, n}}(-1)^{\sigma_{0}(A)} \operatorname{PER}(A)^{n}
$$

where $\sigma_{0}(A)$ is the number of zeroes in $A$. Fu essentially gave (7), but the summation is split in a different way. It seems that [49] and [138] obtained (7) independently as neither paper has mention of the other.

Let $c$ and $d$ be real numbers such that $c \neq 0$ and let $\bar{X}=c X+d J$ where $J$ is the all-1 matrix. It follows that $L_{k, n}$ is the coefficient of $c^{k n} \prod_{i=1}^{k} \prod_{j=1}^{n} x_{i j}$ in $\operatorname{PER}(\bar{X})^{n}$. We claim that

$$
L_{k, n}=c^{-k n} \sum_{A \in \mathcal{B}_{k, n}}(-1)^{\sigma_{0}(A)}\left(\operatorname{PER}(\bar{A})^{n}+f(\operatorname{PER}(\bar{A}))\right)
$$

where $\bar{A}=c A+d J$ and $f$ is any polynomial of degree at most $n-1$. If we let $g=$ $g(A)$ be any summand of $f(\operatorname{PER}(\bar{A}))$ when fully expanded, then $g$ has integral degree in each $a_{i j}$ and total degree at most $k(n-1)$. Therefore $g$ cannot vary with every $a_{i j}$ (otherwise it would have degree at least $k n$ ). Hence $\sum_{A \in \mathcal{B}_{k, n}}(-1)^{\sigma_{0}(A)} g(A)=0$ and so $\sum_{A \in \mathcal{B}_{k, n}}(-1)^{\sigma_{0}(A)} f(\operatorname{PER}(\bar{A}))=0$. 
Equation (8) yields the formula of McKay and Wanless [96] when $c=2, d=-1$ and $k=n$. There were various other formulae for $L_{n}$ and $L_{k, n}$ given by Shao and Wei [138], which are all special cases of (8). There are $2^{k n}$ matrices $A \in \mathcal{B}_{k, n}$ which makes (8) impractical for enumeration.

$\mathrm{Fu}[49]$ also gave the equation

$$
L_{k, n}=\sum_{A \in \mathcal{B}_{n, n}}(-1)^{\sigma_{0}(A)}\left(\begin{array}{c}
n^{2}-k n+\sigma_{0}(A) \\
\sigma_{0}(A)
\end{array}\right) \operatorname{PER}(A)^{k}
$$

which has been rearranged and a problem corrected - the last equation in [49] should have $f_{n(n-r)+k}$ instead of $f_{n(n-r)}$.

Jucys [71] constructed an algebra $\mathcal{A}_{n}$ over $\mathbb{C}$, with the "magic squares" as a basis, which were actually $n \times n$ non-negative integer matrices with row and column sums equal to $n$. Multiplication in $\mathcal{A}_{n}$ was defined using a "structure constant," which, in one case, was $L_{n}$. An isomorphism was identified between $\mathcal{A}_{n}$ and a subalgebra of the group algebra of the symmetric group $S_{n^{2}}$ over $\mathbb{C}$. Representation theory was then used to give an expression for $L_{n}$ in terms of eigenvalues of a particular element of $\mathcal{A}_{n}$.

It seems to us that for obtaining the general formulas for the eigenvalues... some further developments of Young's substitutional analysis are needed.

$$
\text { - Jucys [71] }
$$

Light Jr. [86] (see also [85]) gave an equation for the number of "truncated Latin rectangles" which, for Latin rectangles, simplifies to

$$
L_{k, n}=\sum_{i=0}^{n}(-1)^{i}\left(\begin{array}{l}
n \\
i
\end{array}\right)(n-i) !^{k} a_{k, i, n}
$$

where $a_{k, i, n}$ is the number of $k \times i$ matrices with symbols from a set of cardinality $n$ such that each row does not have a repeated symbol and each column has at least one repeated symbol.

Let $\mathcal{M}_{n}$ be the set of partitions of $n$ into parts of size at least 2 . For $\mu \in \mathcal{M}_{n}$, let $X_{\mu}$ be the number of $2 \times n$ Latin rectangles $L=\left(l_{i j}\right)$ with derangement $l_{0 i} \mapsto l_{1 i}$ having cycle structure $\mu$. In fact

$$
X_{\mu}=\frac{n !^{2}}{\prod_{i}\left(s_{i}(\mu) ! \cdot i^{s_{i}(\mu)}\right)},
$$

where $s_{i}(\mu)$ is the number of copies of $i$ in the partition $\mu$. Theorem 6.2 will show that each $L$ counted by $X_{\mu}$ admits the same number of completions $C_{\mu}$ to a Latin square. Dénes and Mullen [33] gave a formula for $L_{n}$ which is essentially

$$
L_{n}=\sum_{\mu \in \mathcal{M}_{n}} X_{\mu} C_{\mu}
$$


Dénes and Mullen [33] also gave a more general formula, which can be interpreted as partitioning Latin squares into $\lambda_{s} \times n$ Latin rectangles where $n=\lambda_{1}+\lambda_{2}+\cdots+\lambda_{m}$ and counting the number of non-clashing replacements that can be made for each $\lambda_{s} \times n$ Latin rectangle. Equation (9) arises from the case when the partition of $n$ is $2+(n-2)$.

We will now reproduce Doyle's [35] formula for $K_{k, n}$, which he gives for $2 \leqslant k \leqslant 4$. We will consolidate it into a concise general form. Let $\mathcal{R}$ be the set of non-negative integer vectors $\vec{s}=\left(s_{i}\right)_{1 \leqslant i \leqslant 2^{k-1}}$ such that $\sum_{i} s_{i}=n$. For $1 \leqslant i \leqslant 2^{k-1}$, let $\Delta_{i}=\left(\delta_{i j}\right)_{1 \leqslant j \leqslant 2^{k-1}}$, where $\delta_{i j}$ is the Kronecker $\delta$-function. For any non-negative integer $i$ let $b_{j}(i)$ be the $j$-th binary digit of $i$, for example $\left(b_{j}(13)\right)_{j \geqslant 1}=(1,0,1,1,0,0, \ldots)$. Let $\|\vec{s}\|=\sum_{i, j} s_{i} b_{j}(i)$. Then

$$
K_{k, n}=\sum_{\vec{s} \in \mathcal{R}}(-1)^{|| \vec{s}||}\left(\begin{array}{c}
n \\
s_{1}, s_{2}, \ldots, s_{2^{k-1}}
\end{array}\right) \prod_{i=1}^{2^{k-1}} g\left(\vec{s}-\Delta_{i}\right)^{s_{i}}
$$

where subtraction of vectors is component-wise and for $\vec{a}=\left(a_{1}, a_{2}, \ldots, a_{2^{k-1}}\right)$

$$
g(\vec{a})=\sum_{P \in \mathcal{P}_{k-1}} \prod_{p \in P}(-1)^{|p|-1}(|p|-1) ! f_{p}(\vec{a})
$$

where $\mathcal{P}_{k-1}$ is the set of partitions of $\{1,2, \ldots, k-1\}$ and

$$
f_{p}(\vec{a})=\sum_{i: b_{j}(i)=0 \forall j \in p} a_{i}
$$

for all $p \subseteq\{1,2, \ldots, k-1\}$.

The coefficients in (11) were not given by Doyle in full generality, although he did state how to obtain them, that is by Möbius Inversion on the lattice of partitions of $\{1,2, \ldots, k-1\}$ (see [123, p. 360], for example).

The expressions get uglier and uglier at an exponential rate as $k$ increases.

$$
\text { - Doyle [35] }
$$

Now assume $k$ is fixed. The function $g(\vec{a})$ is a $2^{k-1}$-variate polynomial. Therefore the computational complexity of (10) is bounded above by $|\mathcal{R}| h(n) \leqslant n^{2^{k-1}} h(n)=n^{O(1)}$ for some polynomial $h$. According to Wilf [171], the problem of enumerating $k \times n$ Latin rectangles for a fixed $k$ is therefore $p$-solved - there exists an algorithm that returns $R_{k, n}$ in polynomial-time in $n$. Alternatively, we may describe the problem of enumerating $k \times n$ Latin rectangles as fixed parameter tractable.

Wilf arrived at this definition after he refereed a paper proposing a "formula" for the answer to [what is $L_{n}$ ?], and realizing that its "computational complexity" exceeds that of the caveman's formula of direct counting.

- Zeilberger [177] 
Gessel [54] also recognised that $L_{k, n}$, for fixed $k$, is $P$-recursive [145], that is, for fixed $k$, there exists a set of polynomials $c_{i}(n)$ for $0 \leqslant i \leqslant M$ for some finite $M$, such that

$$
\sum_{i=0}^{M} c_{i}(n) L_{k, n+i}=0 .
$$

The author has used (10) to find the values of $R_{4, n}$ for $n \leqslant 80$ (Sloane's [142] A000573), $R_{5, n}$ for $n \leqslant 28$, as listed in Figure 16 and $R_{6, n}$ for $n \leqslant 13$. In particular,

$$
R_{6,12}=16790769154925929673725062021120
$$

and

$$
R_{6,13}=4453330421956050777867897829494620160 \text {. }
$$

We also list $R_{4, n}$ for $4 \leqslant n \leqslant 28$ in Figure 15 . Computing $R_{6, n}$ for $1 \leqslant n \leqslant 13$ took just under two months using a Pentium 4, $3.20 \mathrm{GHz}$ processor. The $\mathrm{C}$ code is available from [152]; it uses the GMP library [60]. The curious prime power divisors $2^{a}$ and $3^{b}$ of $R_{4, n}$ and $R_{5, n}$ are partly explained in [157].

Exact enumeration is difficult for $k>3$.

- Skau [141]

There are some other published formulae for the number of Latin rectangles that will not be given explicitly in this paper because they are similar to (10), in that they found by a combination of Inclusion-Exclusion and Möbius Inversion. These are by Nechvatal [110, 111], Gessel [54] (see also [53]), Athreya, Pranesachar and Singhi [5] and Pranesachar [118]. In a 2007 article, de Gennaro [28] claimed to have found a formula for $R_{k, n}$ and made the following remark.

Until now... no explicit formula is known which permits the calculation of $K_{k, n}$ whatever the value of $k$.

- De Gennaro [28]

This misbelief highlights the need for this survey. Somehow a similar false claim was made by Mullen and Mummert in a 2007 book, despite Mullen (with Dénes) having already published a formula for $L_{n}$ in [33].

As of the writing of this book, no formula for $R_{n}$ has been found and it seems possible that none exists.

- Mullen and Mummert [106, p. 44] 


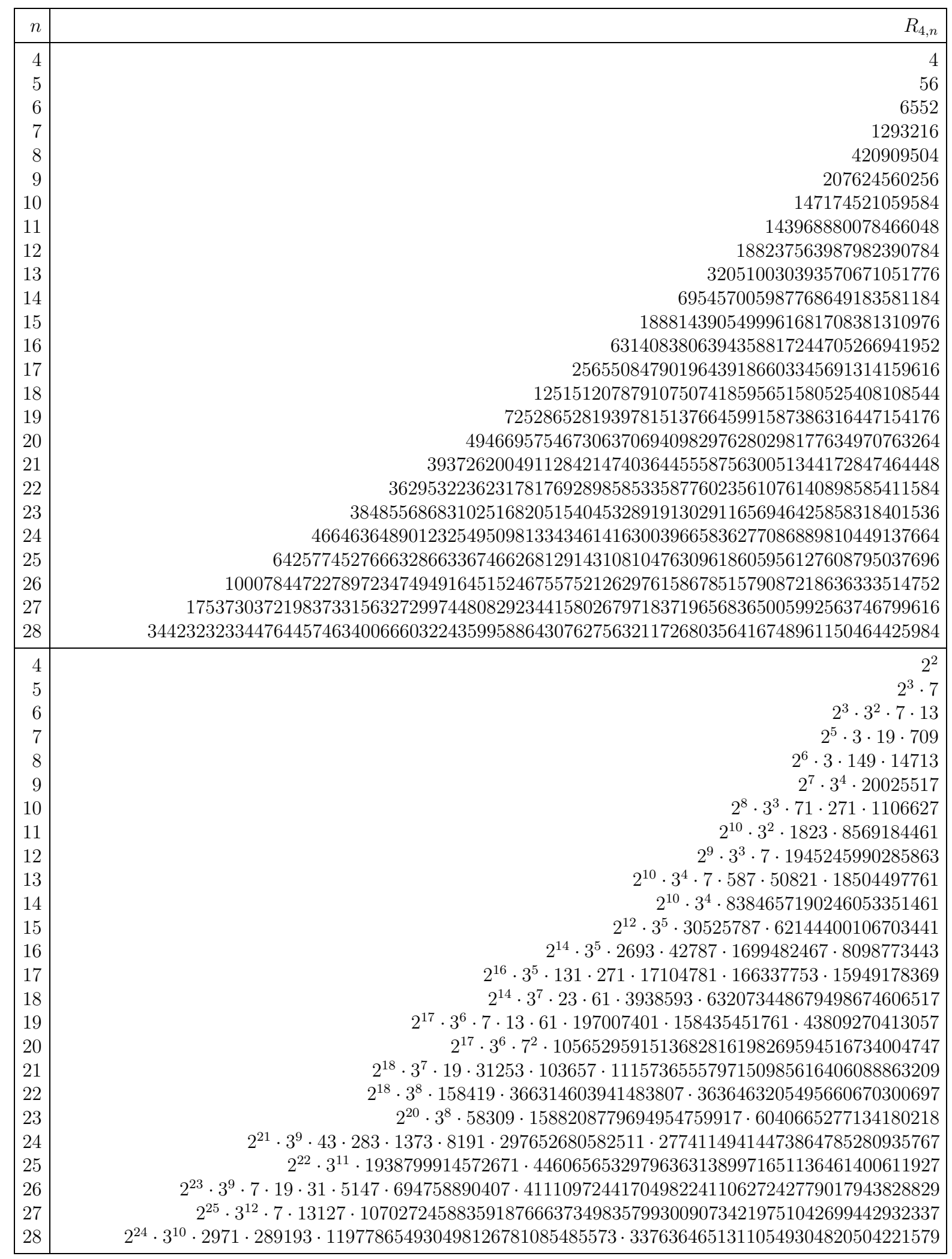

Figure 15: The value of $R_{4, n}$ and its prime factorisation for $4 \leqslant n \leqslant 28$. 


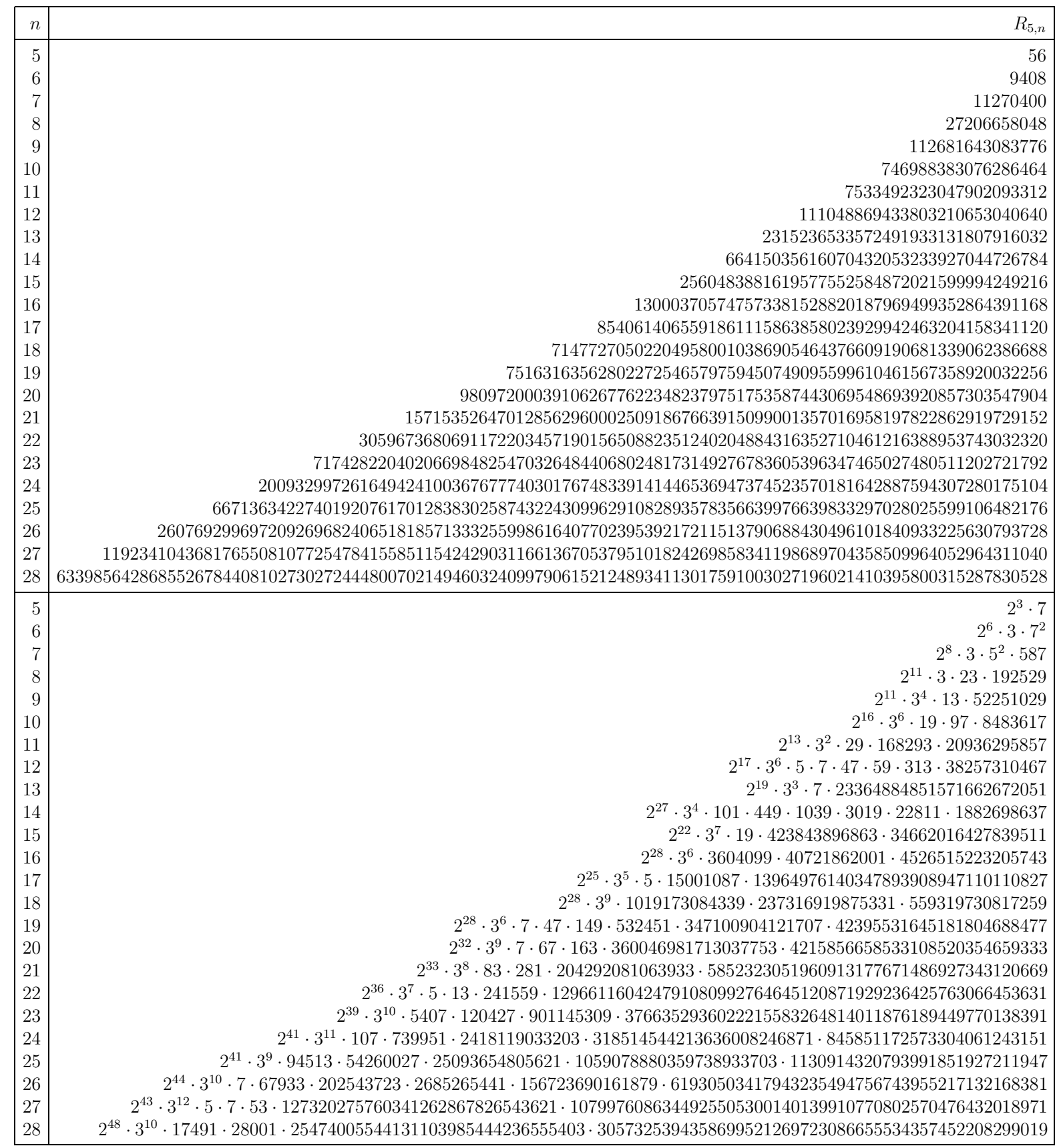

Figure 16: The value of $R_{5, n}$ and its prime factorisation for $5 \leqslant n \leqslant 28$. 
We will now introduce a new formula for $L_{k, n}$ whose complexity lies in computing subgraphs of a given graph. Actually, we arrive at this formula using standard techniques in graph theory [170]. Let $G=G_{k, n}$ be the rook's graph introduced in Section 2.3.1. We identified that $L_{k, n}$ is the number of proper vertex-colourings of $G$ with colour set $\mathbb{Z}_{n}$. Let $E(G)$ be the edge set of $G$. For each non-empty $\mathcal{E} \subseteq E(G)$ let $\mathcal{S}_{\mathcal{E}}$ denote the set of improper vertex-colourings of $G$ such that if $u v \in \mathcal{E}$ then $u$ and $v$ receive the same colour. Let $\mathcal{S}_{\emptyset}$ be the set of all $n^{k n}$ vertex-colourings of $G$. Then

$$
L_{k, n}=\left|\mathcal{S}_{\emptyset}\right|-\left|\bigcup_{\mathcal{E} \subseteq E(G): \mathcal{E} \neq \emptyset} \mathcal{S}_{\mathcal{E}}\right| .
$$

By Inclusion-Exclusion

$$
L_{k, n}=\sum_{\mathcal{E} \subseteq E(G)}(-1)^{|\mathcal{E}|}\left|\mathcal{S}_{\mathcal{E}}\right| .
$$

For any $\mathcal{E} \subseteq E(G)$ let $H_{\mathcal{E}}$ be the graph on the same vertex set as $G$, but with edge set $\mathcal{E}$. Then $\left|\mathcal{S}_{\mathcal{E}}\right|=n^{c\left(H_{\mathcal{E}}\right)}$, where $c\left(H_{\mathcal{E}}\right)$ is the number of connected components of $H_{\mathcal{E}}$. Hence

$$
L_{k, n}=\sum_{\mathcal{E} \subseteq E(G)}(-1)^{|\mathcal{E}|} n^{c\left(H_{\mathcal{E}}\right)} .
$$

There are $|E(G)|=n\left(\begin{array}{c}k \\ 2\end{array}\right)+k\left(\begin{array}{c}n \\ 2\end{array}\right)$ edges in $G$ and $2^{|E(G)|}$ subsets of $E(G)$. While each individual summand of (12) is simple to compute, there are too many terms in the sum for practical use.

For any graph $H$, let $\xi_{k, n}(H)$ be the number of subgraphs of $G_{k, n}$ that are isomorphic to $H$. Let $\Gamma$ be a set of isomorphism class representatives of graphs without isolated vertices; here we include the empty graph in $\Gamma$ which has no vertices, edges and components. Then

$$
L_{k, n}=\sum_{H \in \Gamma}(-1)^{|E(H)|} n^{c(H)+k n-|V(H)|} \xi_{k, n}(H)
$$

where $V(H)$ is the vertex set of $H$ and $E(H)$ is the edge set of $H$. It appears that $\xi_{k, n}$ is a difficult function to compute, thus making this formula for $L_{k, n}$ impractical also. A result of Alon [2] implies that $\xi_{n, n}(H)=O\left(n^{2|V(H)|}\right)$ for any fixed $H \in \Gamma$ as $n \rightarrow \infty$.

\section{Enumeration}

\subsection{Sade's approach}

Modern enumeration algorithms for Latin rectangles, for example those of McKay and Rogoyski [95] and McKay and Wanless [96] (which will be discussed in Section 6.2), stem from a result of Sade [125], which was used to find $R_{7}$. We are motivated to discuss the computation in $[125]$ in detail for the following reasons.

- Sade's approach is still relevant in modern algorithms. 
- Sade's paper [125] is difficult to find and is written in French.

- There is some discrepancy in the literature as to what equivalence relation Sade actually used in [125]; see [173] and [7] vs. [168].

We may say without reserve that Professor Sade was undoubtedly an outstanding mathematician, passionately devoted to mathematical research, and that his work deserves to be thoroughly studied.

- Mitrinović, Janić and Kečkić [103]

\subsubsection{Sade's equivalence.}

Sade's computation was made feasible by the following theorem in [125].

Theorem 6.1. Suppose $L$ is a reduced $k \times n$ Latin rectangle. Suppose we apply all, or some, of the following operations to $L$, to obtain another reduced $k \times n$ Latin rectangle $L^{\prime}$.

1. Permute the columns of $L$;

2. Permute the symbols of $L$;

3. Permute the elements of the columns of $L$ so equal elements do not appear in the same row.

Then $L$ and $L^{\prime}$ can be extended to the same number of reduced Latin squares of order $n$.

The operations described in Theorem 6.1 form an equivalence relation amongst reduced $k \times n$ Latin rectangles such that equivalent Latin rectangles admit the same number of completions to a reduced Latin square of order $n$. Let $\Gamma$ be a set of reduced $k \times n$ Latin rectangles consisting of a representative from each equivalence class. For any $L \in \Gamma$ define $\|L\|$ to be the size of the equivalence class containing $L$ and $\mathrm{C}(L)$ to be the number of completions of $L$ to a reduced Latin square of order $n$. It follows that

$$
R_{n}=\sum_{L \in \Gamma}\|L\| \cdot \mathrm{C}(L)
$$

For instance, when $k=2$ and $n=7$, Sade took $\Gamma$ to contain the following four reduced $2 \times 7$ Latin rectangles (Sade actually used the symbols $1,2, \ldots, 7$, but that is unimportant).

$$
\begin{aligned}
A=\left(\begin{array}{lllllll}
0 & 1 & 2 & 3 & 4 & 5 & 6 \\
1 & 0 & 3 & 2 & 5 & 6 & 4
\end{array}\right), & B=\left(\begin{array}{lllllll}
0 & 1 & 2 & 3 & 4 & 5 & 6 \\
1 & 0 & 3 & 4 & 5 & 6 & 2
\end{array}\right), \\
C=\left(\begin{array}{lllllll}
0 & 1 & 2 & 3 & 4 & 5 & 6 \\
1 & 2 & 3 & 4 & 0 & 6 & 5
\end{array}\right), & D=\left(\begin{array}{lllllll}
0 & 1 & 2 & 3 & 4 & 5 & 6 \\
1 & 2 & 3 & 4 & 5 & 6 & 0
\end{array}\right) .
\end{aligned}
$$

THE ELECTRONIC JOURNAL OF COMBINATORICS 17 (2010), \#A1 
He calculated that $\|A\|=35,\|B\|=84,\|C\|=70$ and $\|D\|=120$. Hence

$$
R_{7}=\left(\begin{array}{llll}
35 & 84 & 70 & 120
\end{array}\right)\left(\begin{array}{l}
\mathrm{C}(A) \\
\mathrm{C}(B) \\
\mathrm{C}(C) \\
\mathrm{C}(D)
\end{array}\right)
$$

Sade found that $A$ and $B$ both admit 116 extensions to a reduced $3 \times 7$ Latin rectangle and $C$ and $D$ admit 115 extensions to a reduced $3 \times 7$ Latin rectangle. He gave a detailed table that partitioned these 462 reduced $3 \times 7$ Latin rectangles into fifteen equivalence classes (using the equivalence relation in Theorem 6.1). The representatives from these equivalence classes of reduced $3 \times 7$ Latin rectangles were called $J, K, M, N, P, Q, \ldots, Z$ and are listed below.

$$
\begin{aligned}
& J=\left(\begin{array}{lllllll}
0 & 1 & 2 & 3 & 4 & 5 & 6 \\
1 & 2 & 3 & 4 & 5 & 6 & 0 \\
2 & 0 & 1 & 5 & 6 & 4 & 3
\end{array}\right) \\
& T=\left(\begin{array}{lllllll}
0 & 1 & 2 & 3 & 4 & 5 & 6 \\
1 & 0 & 3 & 2 & 5 & 6 & 4 \\
2 & 4 & 0 & 5 & 6 & 1 & 3
\end{array}\right) \\
& K=\left(\begin{array}{lllllll}
0 & 1 & 2 & 3 & 4 & 5 & 6 \\
1 & 2 & 3 & 4 & 5 & 6 & 0 \\
2 & 0 & 6 & 5 & 3 & 1 & 4
\end{array}\right) \\
& U=\left(\begin{array}{lllllll}
0 & 1 & 2 & 3 & 4 & 5 & 6 \\
1 & 2 & 3 & 4 & 5 & 6 & 0 \\
2 & 3 & 4 & 5 & 6 & 0 & 1
\end{array}\right) \\
& M=\left(\begin{array}{lllllll}
0 & 1 & 2 & 3 & 4 & 5 & 6 \\
1 & 2 & 3 & 4 & 5 & 6 & 0 \\
2 & 4 & 1 & 0 & 6 & 3 & 5
\end{array}\right) \\
& V=\left(\begin{array}{lllllll}
0 & 1 & 2 & 3 & 4 & 5 & 6 \\
1 & 0 & 3 & 2 & 5 & 6 & 4 \\
2 & 4 & 5 & 6 & 0 & 1 & 3
\end{array}\right) \\
& N=\left(\begin{array}{lllllll}
0 & 1 & 2 & 3 & 4 & 5 & 6 \\
1 & 0 & 3 & 4 & 5 & 6 & 2 \\
2 & 3 & 5 & 6 & 0 & 1 & 4
\end{array}\right) \\
& W=\left(\begin{array}{lllllll}
0 & 1 & 2 & 3 & 4 & 5 & 6 \\
1 & 0 & 3 & 4 & 5 & 6 & 2 \\
2 & 3 & 0 & 5 & 6 & 1 & 4
\end{array}\right) \\
& P=\left(\begin{array}{lllllll}
0 & 1 & 2 & 3 & 4 & 5 & 6 \\
1 & 2 & 3 & 4 & 5 & 6 & 0 \\
2 & 0 & 4 & 1 & 6 & 3 & 5
\end{array}\right) \\
& X=\left(\begin{array}{lllllll}
0 & 1 & 2 & 3 & 4 & 5 & 6 \\
1 & 0 & 3 & 2 & 5 & 6 & 4 \\
2 & 3 & 0 & 1 & 6 & 4 & 5
\end{array}\right) \\
& Q=\left(\begin{array}{lllllll}
0 & 1 & 2 & 3 & 4 & 5 & 6 \\
1 & 0 & 3 & 4 & 5 & 6 & 2 \\
2 & 3 & 5 & 0 & 6 & 4 & 1
\end{array}\right) \\
& Y=\left(\begin{array}{lllllll}
0 & 1 & 2 & 3 & 4 & 5 & 6 \\
1 & 0 & 3 & 2 & 5 & 6 & 4 \\
2 & 3 & 0 & 4 & 6 & 1 & 5
\end{array}\right) \\
& R=\left(\begin{array}{lllllll}
0 & 1 & 2 & 3 & 4 & 5 & 6 \\
1 & 0 & 3 & 4 & 5 & 6 & 2 \\
2 & 3 & 4 & 6 & 0 & 1 & 5
\end{array}\right) \\
& S=\left(\begin{array}{lllllll}
0 & 1 & 2 & 3 & 4 & 5 & 6 \\
1 & 0 & 3 & 4 & 5 & 6 & 2 \\
2 & 3 & 4 & 1 & 6 & 0 & 5
\end{array}\right) \\
& Z=\left(\begin{array}{lllllll}
0 & 1 & 2 & 3 & 4 & 5 & 6 \\
1 & 0 & 3 & 2 & 5 & 6 & 4 \\
2 & 3 & 4 & 5 & 6 & 0 & 1
\end{array}\right)
\end{aligned}
$$

\subsubsection{Sade's invariant.}

Sade distinguished between inequivalent Latin rectangles using an invariant which we will now describe. For each column $C$ he defined a partition $s_{C}$ of $k(k-1)$ where the parts are the number of symbols that appear in both $C$ and $C^{\prime}$, over all columns $C^{\prime} \neq C$ of $L$ (the zero parts are discarded). The multiset $S=S(L)=\left\{s_{C}: C\right.$ is a column of $\left.L\right\}$ is invariant under the operations described in Theorem 6.1. We will call $S(L)$ the Sade invariant. In the case of $k=3$ and $n=7$, Sade labelled the partitions of 6 in the following way. 


$$
\begin{array}{ll}
a: 3+3, & g: 2+2+1+1, \\
b: 3+2+1, & h: 2+1+1+1+1, \\
c: 3+1+1+1, & i: 1+1+1+1+1+1 . \\
d: 2+2+2, &
\end{array}
$$

Then the Sade invariants of the representative reduced $3 \times 7$ Latin rectangles are
$J: 4 b 2 g i$,
$Q: 2 c 2 g 2 h i$
$V: 2 g 4 h i$,
$K: 4 c 3 i$,
$R: 6 h i$,
$W: d 3 g 3 h$,
$M: 6 g i$,
$S: 5 g 2 h$,
$X: 3 a 4 d$
$N: 4 h 3 i$,
$T: 3 g 4 h$,
$Y: 2 b d 3 g h$,
$P: 2 c 3 g 2 h$,
$U: 7 g$,
$Z: g 6 h$

A number before a part indicates its frequency. For example, the Sade invariant of $J$ is $\{b, b, b, b, g, g, i\}$, which is abbreviated to $4 b 2 g i$. Curiously, the Sade invariants for $P$ and $Y$ were listed incorrectly in [125].

\subsubsection{Sade's computation.}

Consequently, it was found that

$$
\left(\begin{array}{c}
\mathrm{C}(A) \\
\mathrm{C}(B) \\
\mathrm{C}(C) \\
\mathrm{C}(D)
\end{array}\right)=\left(\begin{array}{ccccccccccccccc}
\cdot & \cdot & \cdot & \cdot & \cdot & \cdot & \cdot & \cdot & 24 & 24 & 24 & 12 & 2 & 18 & 12 \\
\cdot & \cdot & \cdot & 4 & \cdot & 12 & 8 & 12 & 28 & 4 & 16 & 16 & \cdot & 8 & 8 \\
9 & \cdot & \cdot & 6 & \cdot & 12 & 6 & 12 & 24 & 6 & 24 & \cdot & 1 & 9 & 6 \\
3 & 2 & 2 & 2 & 7 & 14 & 10 & 14 & 16 & 2 & 16 & 8 & \cdot & 11 & 8
\end{array}\right)\left(\begin{array}{c}
\mathrm{C}(J) \\
\mathrm{C}(K) \\
\mathrm{C}(M) \\
\mathrm{C}(N) \\
\mathrm{C}(P) \\
\mathrm{C}(Q) \\
\vdots \\
\mathrm{C}(Z)
\end{array}\right) .
$$

Combined with (13), this yields

$$
\begin{aligned}
R_{7}= & 990 \mathrm{C}(J)+240 \mathrm{C}(K)+240 \mathrm{C}(M)+996 \mathrm{C}(N) \\
& +840 \mathrm{C}(P)+3528 \mathrm{C}(Q)+2292 \mathrm{C}(R)+3528 \mathrm{C}(S) \\
& +6792 \mathrm{C}(T)+1836 \mathrm{C}(U)+5784 \mathrm{C}(V)+2724 \mathrm{C}(W) \\
& +140 \mathrm{C}(X)+3252 \mathrm{C}(Y)+2472 \mathrm{C}(Z) .
\end{aligned}
$$

Sade then computed the number of completions of these fifteen representative reduced $3 \times 7$ Latin rectangles to a reduced Latin square of order 7 . He found 


$$
\begin{aligned}
& \mathrm{C}(J)=\mathrm{C}(W)=\mathrm{C}(Y)=512, \\
& \mathrm{C}(K)=\mathrm{C}(M)=\mathrm{C}(P)=480, \\
& \mathrm{C}(N)=496, \\
& \mathrm{C}(Q)=\mathrm{C}(S)=\mathrm{C}(Z)=464, \\
& \mathrm{C}(R)=\mathrm{C}(V)=472, \\
& \mathrm{C}(T)=\mathrm{C}(U)=456, \\
& \mathrm{C}(X)=576 .
\end{aligned}
$$

Upon substitution into (14), Sade found $R_{7}=16942080$. Sade then attempted to verify his computation as we will now discuss. Sade computed that the number of extensions to a reduced $4 \times 7$ Latin rectangle is

36 for $J, K, M, N, P, R, V$ and $X$,

35 for $T, U$ and $Z$,

34 for $Q$ and $S$,

33 for $W$,

32 for $Y$.

Up to this point, the author has successfully duplicated Sade's enumeration (with the luxury of using GAP [51]). However, in Sade's work some errors arise.

Sade gave another detailed table (containing some errors) that partitioned these 526 reduced $4 \times 7$ Latin rectangles into fifteen equivalence classes (using the equivalence relation in Theorem 6.1). The representatives from these equivalence classes of reduced $4 \times 7$ Latin rectangles were called $j, k, m, n, p, q, \ldots, z$. To correct Sade's "Table d'equivalence des $4^{\text {me }}$ lignes":

- Column $K$ should instead have $s$ whereever $z$ occurs (as noticed by [173]).

- Column $R$ row 17 should be $t$ instead of $v$.

- Column $T$ row 32 should be $v$ instead of $t$.

The corrected table is given in Figure 17. Consequently, it follows that 


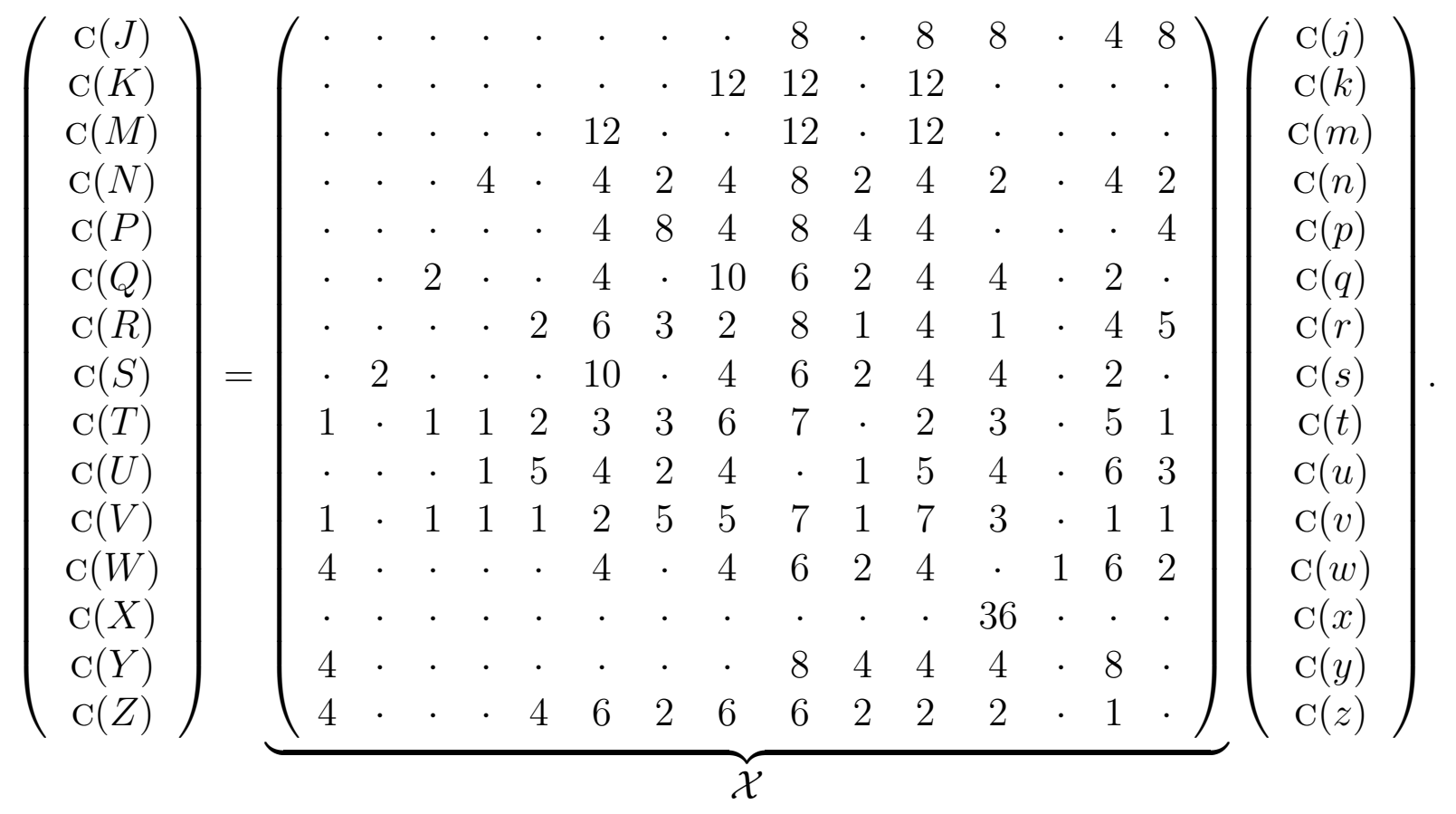

The fifth row of $\mathcal{X}$ was also incorrect in [125], even after correcting for Sade's erroneous version of Figure 17 (although this seems to be merely a typographical error). Combined with (14), this yields

$$
\begin{aligned}
R_{7}= & 46368 \mathrm{C}(j)+7056 \mathrm{C}(k)+19632 \mathrm{C}(m)+18396 \mathrm{C}(n) \\
& +43020 \mathrm{C}(p)+138384 \mathrm{C}(q)+73500 \mathrm{C}(r)+166944 \mathrm{C}(s) \\
& +234264 \mathrm{C}(t)+52776 \mathrm{C}(u)+150516 \mathrm{C}(v)+108492 \mathrm{C}(w) \\
& +2724 \mathrm{C}(x)+126816 \mathrm{C}(y)+48264 \mathrm{C}(z) .
\end{aligned}
$$

Sade then computed the number of completions of these fifteen representative reduced $4 \times 7$ Latin rectangles to a reduced Latin square of order 7 and found the following.

$$
\begin{aligned}
& \mathrm{C}(k)=\mathrm{C}(m)=\mathrm{C}(p)=8 \\
& \mathrm{C}(r)=10 \\
& \mathrm{C}(n)=\mathrm{C}(q)=\mathrm{C}(s)=\mathrm{C}(z)=12, \\
& \mathrm{C}(t)=\mathrm{C}(v)=14 \\
& \mathrm{C}(j)=\mathrm{C}(w)=\mathrm{C}(y)=16 \\
& \mathrm{C}(u)=22 \\
& \mathrm{C}(x)=48
\end{aligned}
$$

Upon substitution into (15), we again find $R_{7}=16942080$. To obtain Sade's erroneous version of (15), we repair only the fifth row of his version of $\mathcal{X}$. Substitution of $\mathrm{C}(j)$, $\mathrm{C}(k)$, etc. into Sade's erroneous version of (15) also gives the value for $R_{7}$. 


\begin{tabular}{c|ccccccccccccccc} 
& $J$ & $K$ & $M$ & $N$ & $P$ & $Q$ & $R$ & $S$ & $T$ & $U$ & $V$ & $W$ & $X$ & $Y$ & $Z$ \\
\hline 1 & $y$ & $v$ & $t$ & $y$ & $r$ & $q$ & $y$ & $q$ & $y$ & $y$ & $w$ & $x$ & $w$ & $j$ & $j$ \\
2 & $y$ & $v$ & $v$ & $y$ & $r$ & $q$ & $y$ & $k$ & $s$ & $q$ & $t$ & $y$ & $w$ & $j$ & $j$ \\
3 & $w$ & $s$ & $t$ & $y$ & $r$ & $q$ & $y$ & $q$ & $t$ & $y$ & $w$ & $y$ & $w$ & $j$ & $p$ \\
4 & $w$ & $t$ & $v$ & $y$ & $r$ & $q$ & $q$ & $q$ & $q$ & $p$ & $t$ & $j$ & $w$ & $j$ & $j$ \\
5 & $w$ & $s$ & $t$ & $q$ & $r$ & $s$ & $q$ & $k$ & $y$ & $q$ & $t$ & $j$ & $w$ & $v$ & $j$ \\
6 & $w$ & $t$ & $v$ & $q$ & $r$ & $s$ & $q$ & $q$ & $w$ & $p$ & $t$ & $y$ & $w$ & $t$ & $p$ \\
7 & $z$ & $t$ & $t$ & $q$ & $r$ & $u$ & $p$ & $v$ & $t$ & $s$ & $r$ & $y$ & $w$ & $v$ & $p$ \\
8 & $z$ & $v$ & $v$ & $q$ & $r$ & $u$ & $q$ & $q$ & $p$ & $p$ & $u$ & $j$ & $w$ & $t$ & $s$ \\
9 & $v$ & $s$ & $q$ & $t$ & $v$ & $y$ & $p$ & $t$ & $q$ & $y$ & $s$ & $z$ & $w$ & $v$ & $s$ \\
10 & $v$ & $t$ & $q$ & $z$ & $s$ & $y$ & $s$ & $v$ & $r$ & $u$ & $q$ & $v$ & $w$ & $t$ & $r$ \\
11 & $v$ & $v$ & $q$ & $u$ & $z$ & $s$ & $q$ & $q$ & $s$ & $v$ & $s$ & $v$ & $w$ & $v$ & $t$ \\
12 & $v$ & $s$ & $q$ & $t$ & $t$ & $s$ & $v$ & $t$ & $t$ & $q$ & $r$ & $s$ & $w$ & $t$ & $s$ \\
13 & $y$ & $s$ & $v$ & $t$ & $q$ & $t$ & $z$ & $t$ & $r$ & $w$ & $s$ & $t$ & $w$ & $y$ & $q$ \\
14 & $y$ & $t$ & $v$ & $w$ & $u$ & $t$ & $z$ & $q$ & $y$ & $y$ & $p$ & $q$ & $w$ & $y$ & $t$ \\
15 & $w$ & $s$ & $t$ & $v$ & $t$ & $m$ & $r$ & $v$ & $w$ & $v$ & $t$ & $t$ & $w$ & $t$ & $v$ \\
16 & $w$ & $t$ & $t$ & $s$ & $z$ & $m$ & $v$ & $s$ & $s$ & $w$ & $n$ & $y$ & $w$ & $u$ & $v$ \\
17 & $w$ & $v$ & $q$ & $v$ & $t$ & $s$ & $t$ & $s$ & $t$ & $z$ & $s$ & $z$ & $w$ & $t$ & $q$ \\
18 & $w$ & $v$ & $v$ & $n$ & $v$ & $s$ & $t$ & $w$ & $n$ & $w$ & $v$ & $t$ & $w$ & $u$ & $t$ \\
19 & $z$ & $v$ & $t$ & $s$ & $s$ & $v$ & $u$ & $y$ & $s$ & $y$ & $y$ & $t$ & $w$ & $y$ & $s$ \\
20 & $z$ & $t$ & $q$ & $s$ & $q$ & $v$ & $t$ & $w$ & $v$ & $n$ & $t$ & $s$ & $w$ & $y$ & $p$ \\
21 & $v$ & $s$ & $v$ & $r$ & $u$ & $w$ & $t$ & $u$ & $r$ & $v$ & $v$ & $u$ & $w$ & $w$ & $s$ \\
22 & $v$ & $v$ & $t$ & $n$ & $t$ & $w$ & $r$ & $q$ & $y$ & $r$ & $j$ & $v$ & $w$ & $w$ & $t$ \\
23 & $v$ & $t$ & $q$ & $t$ & $v$ & $v$ & $t$ & $t$ & $q$ & $v$ & $q$ & $q$ & $w$ & $y$ & $r$ \\
24 & $v$ & $s$ & $q$ & $n$ & $s$ & $v$ & $z$ & $t$ & $p$ & $q$ & $v$ & $j$ & $w$ & $y$ & $s$ \\
25 & $z$ & $s$ & $q$ & $r$ & $z$ & $s$ & $y$ & $q$ & $w$ & $s$ & $t$ & $y$ & $w$ & $t$ & $w$ \\
26 & $z$ & $s$ & $q$ & $t$ & $t$ & $s$ & $q$ & $v$ & $t$ & $v$ & $r$ & $s$ & $w$ & $u$ & $q$ \\
27 & $t$ & $s$ & $v$ & $u$ & $q$ & $t$ & $v$ & $s$ & $z$ & $s$ & $s$ & $v$ & $w$ & $t$ & $u$ \\
28 & $t$ & $s$ & $v$ & $t$ & $u$ & $t$ & $t$ & $s$ & $j$ & $w$ & $w$ & $q$ & $w$ & $u$ & $w$ \\
29 & $t$ & $v$ & $t$ & $t$ & $t$ & $w$ & $t$ & $w$ & $y$ & $y$ & $v$ & $u$ & $w$ & $y$ & $t$ \\
30 & $t$ & $v$ & $t$ & $t$ & $z$ & $w$ & $z$ & $y$ & $m$ & $r$ & $r$ & $s$ & $w$ & $y$ & $t$ \\
31 & $z$ & $t$ & $q$ & $z$ & $t$ & $t$ & $v$ & $w$ & $s$ & $s$ & $v$ & $t$ & $w$ & $w$ & $q$ \\
32 & $z$ & $t$ & $v$ & $v$ & $v$ & $t$ & $z$ & $u$ & $v$ & $z$ & $r$ & $t$ & $w$ & $w$ & $u$ \\
33 & $t$ & $v$ & $t$ & $w$ & $s$ & $s$ & $w$ & $q$ & $s$ & $z$ & $z$ & $q$ & $w$ & $\cdot$ & $q$ \\
34 & $t$ & $t$ & $q$ & $s$ & $q$ & $s$ & $t$ & $t$ & $t$ & $p$ & $m$ & $\cdot$ & $w$ & $\cdot$ & $q$ \\
35 & $t$ & $v$ & $v$ & $n$ & $u$ & $\cdot$ & $s$ & $\cdot$ & $t$ & $p$ & $v$ &. & $w$ &. & $y$ \\
36 & $t$ & $t$ & $t$ & $v$ & $t$ & $\cdot$ & $r$ &. & $\cdot$ & $\cdot$ & $v$ &. & $w$ &. &.
\end{tabular}

Figure 17: The corrected version of Sade's table, which partitions the extensions of $J, K, M, N, P, Q, \ldots, Z$ into the equivalence classes $j, k, m, n, p, q, \ldots, z$. 


\subsubsection{Discrepancy.}

As mentioned earlier, there is some discrepancy in the literature as to which equivalence relation Sade used in [125], which we will now discuss.

Recall the definition of the template of a Latin rectangle from Section 3. We call two $k \times n$ Latin rectangles $L$ and $L^{\prime}$ template equivalent if the template of $L^{\prime}$ can be formed from the template $T$ of $L$, or the transpose of $T$, by some row and column permutations.

Theorem 6.2. Suppose $L$ and $L^{\prime}$ are template equivalent $k \times n$ Latin rectangles. Then for any $k^{*}$ in the range $k \leqslant k^{*} \leqslant n$, it follows that $L$ and $L^{\prime}$ can be extended to the same number of $k^{*} \times n$ Latin rectangles. Moreover, if $L$ and $L^{\prime}$ are both reduced, then $L$ and $L^{\prime}$ can be completed to the same number of reduced Latin squares.

Proof. Let $T=\left(t_{i j}\right)$ be the template of $L$. From $T$ we can construct a bipartite graph $G=G(L)$ with bipartition $\left\{u_{0}, u_{1}, \ldots, u_{n-1}\right\} \cup\left\{w_{0}, w_{1}, \ldots, w_{n-1}\right\}$ and an edge from $u_{i}$ to $w_{j}$ whenever $t_{i j}=1$. Since $L$ and $L^{\prime}$ are template equivalent, $G(L)$ is isomorphic to $G\left(L^{\prime}\right)$. See Section 2.3.3 for more details about the equivalence between Latin squares and one-factorisations of balanced complete bipartite graphs.

The number of extensions of $L$ (or $L^{\prime}$ ) to a $k^{*} \times n$ Latin rectangle is the number of ordered $\left(k^{*}-k\right)$-tuples of disjoint one-factors in $G(L) \cong G\left(L^{\prime}\right)$, thus proving the first claim in the theorem. The number of completions of $L$ (or $L^{\prime}$ ) to a Latin square is therefore equal to the number of one-factorisations of $G(L)$. The number of completions of $L$ (or $\left.L^{\prime}\right)$ to a Latin square is $(n-k)$ ! times the number of completions of $L$ to a reduced Latin square.

The first statement in Theorem 6.2 is not true if we restrict to reduced Latin rectangles. For example, the template equivalent reduced $2 \times 5$ Latin rectangles

$$
\left(\begin{array}{lllll}
0 & 1 & 2 & 3 & 4 \\
1 & 2 & 3 & 4 & 0
\end{array}\right) \text { and } \quad\left(\begin{array}{lllll}
0 & 1 & 2 & 3 & 4 \\
1 & 3 & 4 & 2 & 0
\end{array}\right)
$$

admit 4 and 5 extensions to a reduced $3 \times 5$ Latin rectangle, respectively. Hence, if we use Sade's approach to compute $R_{n}$, then the numbers that arise during the intermediate steps in the computation (such as (13), etc. or as in [169, Fig. 7.6]) will vary depending on which equivalence class representatives are chosen.

To illustrate the difference between Theorems 6.1 and 6.2 , if we let

$$
L=\left(\begin{array}{lllllll}
0 & 1 & 2 & 3 & 4 & 5 & 6 \\
1 & 0 & 3 & 4 & 5 & 6 & 2 \\
2 & 6 & 0 & 5 & 3 & 1 & 4
\end{array}\right)
$$

then

$$
L^{(c s)}=\left(\begin{array}{ccccccc}
0 & 1 & 2 & 3 & 4 & 5 & 6 \\
1 & 0 & 6 & 2 & 3 & 4 & 5 \\
2 & 5 & 0 & 4 & 6 & 3 & 1
\end{array}\right)
$$

is the Latin rectangle obtained by replacing each row of $L$, when considered as a permutation of $\mathbb{Z}_{7}$, by its inverse. So the template $T(L)$ is the transpose of $T\left(L^{(c s)}\right)$. However, 
the Sade invariant of $L$ is $2 c 2 g 2 h i$ whereas the Sade invariant of $L^{(c s)}$ is $5 g 2 h$. Therefore, $L$ and $L^{(c s)}$ are not equivalent in the sense of Theorem 6.1, while they are equivalent in the sense of Theorem 6.2.

As per our discussion at the start of this section, we note that Yamamoto [173] and Bammel and Rothstein [7] describe Sade's algorithm accurately, while the description given by Wells [168] (and consequently [29, pp. 142-143]) was inaccurate. Specifically, Sade used template equivalence, but without transposition, whereas [168] suggested that Sade used isotopism equivalence. However, Wells later gave an accurate description of Sade's algorithm in his book [169, pp. 204-205] illustrated with the case $n=5$.

In 1948, Sade [125] enumerated reduced $7 \times 7$ squares by a method which by-passes the construction of species representatives. (His total of 16,942,080, however, did lead to the discovery of the 147th species [127] which had been overlooked by Norton [114].) Sade's method is to successively calculate for $k=1,2, \ldots, K, K \leqslant n$, a (complete) set of reduced $k \times n$ Latin rectangles... inequivalent under row, column or label permutation, keeping track of the number of ways each rectangle could have been formed. The $(k+1)$-row rectangles are formed by adding a row to each $k$-row rectangle in all possible ways, eliminating equivalent rectangles (actually the difficult part of the calculation) as they appear. When $k=K$ (Sade used $K=4$ ), one may sum the product of the number of ways in which a rectangle could have been formed (already known) and the number of ways the rectangle can be completed to a square (easily computed) over the inequivalent $K$-row rectangles, producing the number of $n \times n$ squares.

- Wells [168]

Sade's method is based on the fact that equivalent Latin rectangles can be filled out to complete $n \times n$ Latin squares in the same number of ways (have the same count) where equivalent Latin rectangles are intertransformable by column and label permutations such that corresponding columns have the same set of (unordered) symbols.

- Bammel and Rothstein [7]

\subsection{McKay and Wanless' approach}

Sade's idea was first adapted to the computer by Wells [168] (see also [169]), who gave the correct value for $R_{8}$, which he computed on the MANIAC II computer at the Los Alamos Scientific Laboratory. Bammel and Rothstein [7] verified the values for $R_{7}$ and $R_{8}$ and discovered $R_{9}$, with the use of the PDP-10 (Programmed Data Processor model 10) computer. The algorithms of McKay and Rogoyski [95] and McKay and Wanless [96] which were used to find $R_{10}$ and $R_{11}$, respectively, were graph theoretic adaptations of Theorem 6.2 that made use of nauty [93]. 
We will now describe the formulae that McKay and Wanless [96] used to find $R_{k, 11}$. Given a $k \times n$ Latin rectangle $L$, we can construct a bipartite graph $B=B(L)$ with vertex bipartition $C \cup S$ where $C$ is the set of columns of $L$, and $S$ is the set of symbols of $L$. Vertices $c \in C$ and $s \in S$ are adjacent if and only if symbol $s$ occurs in column $c$ in $L$. So $B$ is regular of degree $k$.

Let $\mathcal{T}$ be the group of paratopisms that combines isotopisms of the form $(\varepsilon, \beta, \gamma)$ and (cs)-conjugation, where $\varepsilon$ is the identity permutation. Then $\mathcal{T}$ acts on the set of $k$-regular bipartite graphs $B$, by permuting the vertices of $C$ and $S$ individually and possibly by swapping the sets $C$ and $S$. Let $\mathcal{B}(k, n)$ be a set containing one representative from each orbit of $k$-regular bipartite graphs with vertex bipartition $C \cup S$ under the action of $\mathcal{T}$. For any $B \in \mathcal{B}(k, n)$ let $\operatorname{Aut}_{\mathcal{T}}(B)$ denote the group of all $\tau \in \mathcal{T}$ such that $\tau(B)=B$.

Let $B^{\prime}$ be the bipartite complement of $B$, that is, $B^{\prime}$ is a bipartite graph with vertex bipartition $C \cup S$ such that $c \in C$ is adjacent to $s \in S$ if and only if symbol $s$ does not occur in column $c$ in $L$. In fact, the biadjacency matrix of $B^{\prime}$ is the template of $L$ (see Section 3). Recall that, for any graph, a one-factor is a 1-regular spanning subgraph and a one-factorisation is a decomposition into one-factors. Let $m(H)$ denote the number of one-factorisations of a graph $H$. Whenever $0 \leqslant k \leqslant n$ and $n \geqslant 1$,

$$
R_{n}=2 n k !(n-k) ! \sum_{B \in \mathcal{B}(k, n)} \frac{m(B) m\left(B^{\prime}\right)}{\left|\operatorname{Aut}_{\mathcal{T}}(B)\right|}
$$

and

$$
R_{k, n}=2 n k !(n-k) ! \sum_{B \in \mathcal{B}(k, n)} \frac{m(B)}{\left|\operatorname{Aut}_{\mathcal{T}}(B)\right|} .
$$

It is possible to compute $m(B)$ with the recurrence relation

$$
m(B)=\sum_{F \in \mathcal{F}_{e}} m(B-F)
$$

for any edge $e$ of $B$, where $\mathcal{F}_{e}$ is the set of all one-factors $F$ of $B$ that contain $e$. We use $B-F$ to denote the graph formed by deleting the edges from $B$ that are also in $F$. Describing their computation of $R_{k, 11}$, McKay and Wanless [96] made the following comment.

The main practical difficulty was the efficient management of the fairly large amount of data.

- McKay and Wanless [96]

\section{The asymptotic number of Latin rectangles}

In this section we will survey the asymptotic number of Latin rectangles. We know $L_{1, n}=n$ ! and $L_{2, n} \sim n !^{2} \exp (-1)$ by (5). Kerawala [76] proved that $L_{3, n} \sim n !^{3} c$ for some constant $c \approx \exp (-3)$. Riordan [120] subsequently showed that $L_{3, n} \sim n !^{3} \exp (-3)$ and made the following remark. 
.... a result which Kerawala surmised but failed to prove, though his numerical evidence was practically conclusive (agreeing with $\exp (-3)$ to seven decimal places).

Erdős and Kaplansky [40] found that

$$
L_{k, n} \sim n !^{k} \exp (-k(k-1) / 2)
$$

for $k=O\left((\log n)^{3 / 2-\epsilon}\right)$ and conjectured that (16) is valid for $o\left(n^{1 / 3}\right)$ which was later proved by Yamamoto [172]. Eighteen years later, Yamamoto [175] proved that

$$
L_{k, n} \sim n !^{k} \exp \left(-k(k-1) / 2-k^{3} / 6 n\right)
$$

for $k=O\left(n^{5 / 12-\epsilon}\right)$. Stein [146] (see also [147, 148]) showed that (17) is valid for $o\left(n^{1 / 2}\right)$. Godsil and McKay [58] (see also [57]) proved

$$
L_{k, n} \sim n !^{k}\left(n(n-1) \cdots(n-k+1) / n^{k}\right)^{n}(1-k / n)^{-n / 2} \exp (-k / 2)
$$

as $n \rightarrow \infty$ with $k=o\left(n^{6 / 7}\right)$. Nechvatal [111] and Green [61,62,63] studied the asymptotic number of a generalisation of Latin rectangles.

Comtet [26, p. 183] said that even estimating $L_{n}$ when $n \rightarrow \infty$ "seems to be an extremely difficult combinatorial problem." However, van Lint and Wilson [163, p. 162] showed that

$$
\frac{1}{n} L_{n}^{1 / n^{2}} \rightarrow \exp (-2)
$$

which was conjectured earlier in [115]. This is not a particularly satisfying result since, for example, (2) and Stirling's Approximation imply

$$
\lim _{n \rightarrow \infty} \frac{1}{n} R_{n}^{1 / n^{2}}=\lim _{n \rightarrow \infty} \frac{1}{n} L_{n}^{1 / n^{2}}=\exp (-2),
$$

despite $L_{n}$ and $R_{n}$ differing by a factor of $n !(n-1)$ !. Skau [141] extended (18) to

$$
L_{n}^{1 / n^{1+\epsilon}} \sim n^{n^{1-\epsilon}} \exp \left(-2 n^{1-\epsilon}\right)
$$

for all $\epsilon>0$.

...there is still a long way to go to achieve a 'clean' asymptotic solution for the number of Latin squares.

- Skau [141]

Timashov [160] made the following conjecture.

\section{Conjecture 7.1.}

$$
R_{n} \sim \frac{1}{2}(2 \pi)^{3 n / 2} \exp \left(-2 n^{2}+3 n / 2-1\right) n^{n^{2}-n / 2-1} .
$$


Conjecture 7.1 corresponds well with the estimates in Figure 2, most of which were published after Timashov made Conjecture 7.1. For example, Figure 2 lists the estimates $R_{50} \approx 3.06 \times 10^{2123}$ and $R_{100} \approx 1.78 \times 10^{11396}$ by Zhang and Ma [178], whereas the righthand side of (19) is approximately $3.02 \times 10^{2123}$ and $1.76 \times 10^{11396}$ when $n=50$ and $n=100$, respectively.

\section{Concluding remarks}

In this paper we have identified numerous formulae involving the number of Latin rectangles $L_{k, n}$. Interest in Latin rectangles and their generalisations is largely due to their connection with Latin squares.

The general formulae in Section 5 appear unable to help us find unknown values of $L_{n}$. McKay et al. $[94,95,96]$ gave practical formulae for the enumeration of Latin squares that can be used for computer enumeration, all of which are particularly suited for the use of nauty [93]. However, these formulae are unable to find $R_{12}$ in a reasonable amount of time with current hardware. Since $R_{12} \approx 1.62 \cdot 10^{44}>3 \cdot 10^{10} \cdot R_{11}$, the evaluation of $R_{12}$ is likely to remain infeasible for some years yet.

It appears likely that formulae for the number of Latin squares will continue to be discovered. Although these formulae may not help find unknown values of $R_{n}$ they might be able to shed some light on related problems. For example, they could potentially aid the search for the asymptotic value of $R_{n}$ or help the discovery of divisors of $R_{n}$.

\section{Acknowledgements}

The author would like to thank Ian Wanless and Graham Farr for valuable feedback. Thanks to Donald Keedwell for some historical remarks. Thanks also to Brendan McKay, Richard Brualdi, Xiaoguang Liu, Gang Wang and Arthur Yang for assistance tracking down references.

The author would like to thank the anonymous referee for excellent feedback.

\section{References}

[1] A. A. Albert, Quasigroups II, Trans. Amer. Math. Soc., 55 (1944), pp. 401-419.

[2] N. Alon, On the number of subgraphs of prescribed type of graphs with a given number of edges, Israel J. Math., 38 (1981), pp. 116-130.

[3] R. Alter, How many Latin squares are there?, Amer. Math. Monthly, 82 (1975), pp. 632-634.

[4] V. L. Arlazarov, A. M. Baraev, J. U. Gol'fand and I. A. Faradžev, Construction with the aid of a computer of all Latin squares of order 8, Algorithmic Investigations in Combinatorics, (1978), pp. 129-141. In Russian. 
[5] K. B. Athreya, C. R. Pranesachar and N. M. Singhi, On the number of Latin rectangles and chromatic polynomial of $L\left(K_{r, s}\right)$, European J. Combin., 1 (1980), pp. 9-17.

[6] R. A. Bailey and P. J. Cameron, Latin squares: Equivalents and equivalence, (2003). http://designtheory.org/library/encyc/latinsq/g/topics/lsee.pdf

[7] S. E. Bammel and J. Rothstein, The number of $9 \times 9$ Latin squares, Discrete Math., 11 (1975), pp. 93-95.

[8] R. C. Bose and B. Manvel, Introduction to Combinatorial Theory, Wiley, 1984.

[9] L. J. Brant and G. L. Mullen, A note on isomorphism classes of reduced Latin squares of order 7, Util. Math., 27 (1985), pp. 261-263.

[10] L. M. BRÈGman, Certain properties of nonnegative matrices and their permanents, Soviet Math. Dokl., 14 (1973), pp. 945-949.

[11] J. W. Brown, Enumeration of Latin Squares and Isomorphism Detection in Finite Planes, PhD thesis, University of Los Angeles, 1966.

[12] _ Enumeration of Latin squares with application to order 8, J. Combin. Theory, 5 (1968), pp. 177-184.

[13] R. H. Bruck, Simple quasigroups, Bull. Amer. Math. Soc., 50 (1944), pp. 769-781.

[14] _ Some results in the theory of quasigroups, Trans. Amer. Math. Soc., 55 (1944), pp. 19-52.

[15] R. H. Bruck, Finite nets. I. Numerical invariants, Canadian J. Math., 3 (1951), pp. 94-107.

[16] —_, Finite nets. II. Uniqueness and imbedding, Pacific J. Math., 13 (1963), pp. $421-457$.

[17] B. F. Bryant and H. Schneider, Principal loop-isotopes of quasigroups, Canad. J. Math., 18 (1966), pp. 120-125.

[18] D. Bryant, B. M. Maenhaut, and I. M. Wanless, a family of perfect factorisations of complete bipartite graphs, J. Combin. Theory Ser. A, 98 (2002), pp. 328 342.

[19] _ New families of atomic Latin squares and perfect 1-factorisations, J. Combin. Theory Ser. A, 113 (2004), pp. 608-624.

[20] L. Carlitz, Congruences connected with three-line Latin rectangles, Proc. Amer. Math. Soc., 4 (1953), pp. 9-11.

[21] A. Cayley, On Latin squares, Messenger of Math., 19 (1890), pp. 135-137. See [22], vol. 13 , no. 903 , pp. 55-57.

[22] — The Collected Mathematical Papers of Arthur Cayley, Cambridge University Press, 1897.

[23] G.-S. Cheon and I. M. Wanless, An update on Minc's survey of open problems involving permanents, Linear Algebra Appl., 403 (2005), pp. 314-342. 
[24] C. J. Colbourn, The complexity of completing partial Latin squares, Discrete Appl. Math., 8 (1984), pp. 25-30.

[25] C. J. Colbourn, J. H. Dinitz, et Al., The CRC Handbook of Combinatorial Designs, CRC Press, 1996.

[26] L. Comtet, Advanced Combinatorics: The Art of Finite and Infinite Expansions, Reidel, 1974.

[27] P. Csörgő, A. DrÁpAl AND M. K. Kinyon, Buchsteiner loops, (2007). arXiv:0708.2358v1 [math.GR].

[28] A. De Gennaro, How many Latin rectangles are there?, (2007). arXiv:0711.0527v1 [math.CO].

[29] J. DÉnes And A. D. Keedwell, Latin Squares and their Applications, Academic Press, 1974.

[30] — Latin squares and 1-factorizations of complete graphs. I. Connections between the enumeration of Latin squares and rectangles and $r$-factorizations of labelled graphs, Ars Combin., 25 (1988), pp. 109-126.

[31] _ Latin squares and one-factorizations of complete graphs. II. Enumerating onefactorizations of the complete directed graph $K_{n}^{*}$ using MacMahon's double partition idea, Util. Math., 34 (1988), pp. 73-83.

[32] — Latin Squares: New Developments in the Theory and Applications, NorthHolland, 1991.

[33] J. DÉnes AND G. L. Mullen, Enumeration formulas for Latin and frequency squares, Discrete Math., 111 (1993), pp. 157-163.

[34] J. H. Dinitz And D. R. Stinson, Contemporary Design Theory: A Collection of Surveys, Wiley, 1992.

[35] P. G. Doyle, The number of Latin rectangles, (2007). arXiv:math/0703896v1 [math.CO].

[36] A. A. Drisko, On the number of even and odd Latin squares of order $p+1$, Adv. Math., 128 (1997), pp. 20-35.

[37] —, Proof of the Alon-Tarsi Conjecture for $n=2^{r} p$, Electron. J. Combin., 5 (1998). R28, 5 pp.

[38] L. DuAN, An upper bound for the number of normalized Latin square, Chinese Quart. J. Math., 21 (2006), pp. 585-589.

[39] G. P. EGORICHEv, The solution of the van der Waerden problem for permanents, Adv. Math., 42 (1981), pp. 299-305.

[40] P. ERDös AND I. KAPlAnsky, The asymptotic number of Latin rectangles, Amer. J. Math., 68 (1946), pp. 230-236.

[41] M. J. ERickson, Introduction to Combinatorics, Wiley, 1996.

[42] I. M. H. Etherington, Transposed algebras, Proc. Edinburgh Math. Soc. (2), 7 (1945), pp. 104-121. 
[43] L. EulER, Recherches sur une nouvelle espéce de quarrés magiques, Verh. Zeeuwsch. Gennot. Weten. Vliss., 9 (1782), pp. 85-239. Eneström E530, Opera Omnia OI7, pp. 291-392.

[44] R. M. FALCón, Cycle structures of autotopisms of the Latin squares of order up to 11, Ars Combin., (2009). To appear.

[45] R. M. Falcón and J. Martín-Morales, Gröbner bases and the number of Latin squares related to autotopisms of order $\leqslant 7$, J. Symbolic Comput., 42 (2007), pp. $1142-1154$.

[46] D. I. Falikman, A proof of van der Waerden's conjecture on the permanent of a doubly stochastic matrix, Math. Notes, 29 (1981), pp. 475-479.

[47] R. A. Fisher and F. Yates, The $6 \times 6$ Latin squares, Proc. Cambridge Philos. Soc., 30 (1934), pp. 492-507.

[48] M. Frolov, Sur les permutations carrés, J. de Math. spéc., 4 (1890), pp. 8-11 and 25-30.

[49] Z.-L. Fu, The number of Latin rectangles, Math. Practice Theory, (1992), pp. 4041. In Chinese.

[50] R. M. F. Ganfornina, Latin squares associated to principal autotopisms of long cycles. Application in cryptography, in Proceedings of Transgressive Computing, 2006, pp. 213-230.

[51] The GAP Group, GAP - Groups, algorithms, programming - A system for computational discrete algebra. http://www.gap-system.org/

[52] P. Garcia, Major Percy Alexander MacMahon, PhD thesis, Open University, 2006.

[53] I. M. Gessel, Counting three-line Latin rectangles, in Proceedings of the Colloque de Combinatoire Énumérative, Springer, 1986.

[54] _ Counting Latin rectangles, Bull. Amer. Math. Soc., 16 (1987), pp. 79-83.

[55] S. G. Ghurye, A characteristic of species of $7 \times 7$ Latin squares, Ann. Eugenics, 14 (1948), p. 133.

[56] D. G. GLynn, The conjectures of Alon-Tarsi and Rota in dimension prime minus one, SIAM J. Discrete Math., 24 (2010), pp. 394-399.

[57] C. D. Godsil And B. D. McKay, Asymptotic enumeration of Latin rectangles, Bull. Amer. Math. Soc. (N.S.), 10 (1984), pp. 91-92.

[58] _ Asymptotic enumeration of Latin rectangles, J. Combin. Theory Ser. B, 48 (1990), pp. 19-44.

[59] I. P. Goulden and D. M. Jackson, Combinatorial Enumeration, Wiley, 1983.

[60] T. Granlund ET AL., GMP - GNU multiple precision arithmetic library. http://gmplib.org/

[61] T. A. Green, Asymptotic Enumeration of Latin Rectangles and Associated Waiting Times, PhD thesis, Stanford University, 1984. 
[62] _ Asymptotic enumeration of generalized Latin rectangles, J. Combin. Theory Ser. A, 51 (1989), pp. 149-160.

[63] — Erratum: "Asymptotic enumeration of generalized Latin rectangles", J. Combin. Theory Ser. A, 52 (1989), p. 322.

[64] L. Habsieger And J. C. M. JAnssen, The difference in even and odd Latin rectangles for small cases, Ann. Sci. Math. Québec, 19 (1995), pp. 69-77.

[65] M. Hall, JR., Distinct representatives of subsets, Bull. Amer. Math. Soc., 54 (1948), pp. 922-926.

[66] C. Hämälä̈Inen And N. J. CAvenagh, Transitive Latin bitrades, (2008). arXiv:0804.4663v1 [math.CO].

[67] J. R. Hamilton and G. L. Mullen, How many $i-j$ reduced Latin rectangles are there?, Amer. Math. Monthly, 87 (1980), pp. 392-394.

[68] A. Heinze AND M. KLIN, Links between Latin squares, nets, graphs and groups: Work inspired by a paper of A. Barlotti and K. Strambach, Electron. Notes Discrete Math., 23 (2005), pp. 13-21.

[69] A. Hulpke, P. Kaski And P. R. J. ÖstergÅRD, The number of Latin squares of order 11, Math. Comp. To appear.

[70] S. M. JACOB, The enumeration of the Latin rectangle of depth three by means of a formula of reduction, with other theorems relating to non-clashing substitutions and Latin squares, Proc. London Math. Soc. (2), 31 (1930), pp. 329-354.

[71] A.-A. A. JuCYs, The number of distinct Latin squares as a group-theoretical constant, J. Combin. Theory Ser. A, 20 (1976), pp. 265-272.

[72] D. JungniCKeL, Latin squares, their geometries and their groups. A survey, IMA Vol. Math. Appl., 21 (1990), pp. 166-225.

[73] W. B. JuRkat And H. J. RYser, Matrix factorizations of determinants and permanents, J. Algebra, 3 (1966), pp. 1-27.

[74] V. Kala And A. D. Keedwell, Addendum to "The existence of Buchsteiner and conjugacy-closed quasigroups", European J. Combin., 30 (2009), p. 1386.

[75] A. D. KeEDWELL, The existence of Buchsteiner and conjugacy-closed quasigroups, European J. Combin., 30 (2009), pp. 1382-1385.

[76] S. M. KeraWALA, The enumeration of the Latin rectangle of depth three by means of a difference equation, Bull. Calcutta Math. Soc., 33 (1941), pp. 119-127.

[77] B. Kerby And J. D. H. Smith, Quasigroup automorphisms and symmetric group characters, Comment. Math. Univ. Carol., 51 (2010), pp. 279-286.

[78] —, Quasigroup automorphisms and the Norton-Stein complex, Proc. Amer. Math. Soc. To appear.

[79] M. K. Kinyon, K. Kunen and J. D. Phillips, Diassociativity in conjugacy closed loops, Comm.. Algebra, 32 (2004), pp. 767-786. 
[80] D. Klyve And L. Stemkoski, Graeco-Latin squares and a mistaken conjecture of Euler, Cambridge Mathematical Journal, 37 (2006), pp. 2-15.

[81] G. Kolesova, C. W. H. Lam and L. Thiel, On the number of $8 \times 8$ Latin squares, J. Combin. Theory Ser. A, 54 (1990), pp. 143-148.

[82] N. Y. Kuznetsov, Estimating the number of Latin rectangles by the fast simulation method, Cybernet. Systems Anal., 45 (2009), pp. 69-75.

[83] C. Laywine And G. L. Mullen, Discrete Mathematics using Latin Squares, Wiley, 1998.

[84] H. LiAng And F. BAI, An upper bound for the permanent of $(0,1)$-matrices, Linear Algebra Appl., 377 (2004), pp. 291-295.

[85] F. W. Light, JR., A procedure for the enumeration of $4 \times n$ Latin rectangles, Fibonacci Quart., 11 (1973), pp. 241-246.

[86] — Enumeration of truncated Latin rectangles, Fibonacci Quart., 17 (1979), pp. 34-36.

[87] P. A. Macmahon, A new method in combinatory analysis, with applications to Latin squares and associated questions, Trans. Camb. Phil. Soc., 16 (1898), pp. 262290.

[88] — Combinatorial analysis. the foundations of a new theory, Philos. Trans. Roy. Soc. London Ser. A, 194 (1900), pp. 361-386.

[89] — Combinatory Analysis, Chelsea, 1960.

[90] —-, Percy Alexander MacMahon : collected papers / edited by George E. Andrews, MIT Press, 1986.

[91] B. M. Maenhaut And I. M. Wanless, Atomic Latin squares of order eleven, J. Combin. Des., 12 (2004), pp. 12-34.

[92] M. MArcus, Henryk Minc - a biography, Linear Multilinear Algebra, 51 (2003), pp. $1-10$.

[93] B. D. MCKAY, nauty - Graph isomorphism. http://cs.anu.edu.au/ bdm/nauty/

[94] B. D. McKay, A. Meynert And W. Myrvold, Small Latin squares, quasigroups, and loops, J. Combin. Des., 15 (2007), pp. 98-119.

[95] B. D. McKay And E. Rogoyski, Latin squares of order ten, Electron. J. Combin., 2 (1995). N3, 4 pp.

[96] B. D. MCKay And I. M. Wanless, On the number of Latin squares, Ann. Comb., 9 (2005), pp. 335-344.

[97] H. Minc, Upper bounds for permanents of $(0,1)$-matrices, Bull. Amer. Math. Soc., 69 (1963), pp. 789-791.

[98] —, An inequality for permanents of $(0,1)$-matrices, J. Combinatorial Theory, 2 (1967), pp. 321-326.

[99] — On lower bounds for permanents of $(0,1)$ matrices, Proc. Amer. Math. Soc., 22 (1969), pp. 117-123. 
[100] — Permanents, Addison-Wesley, 1978.

[101] — Theory of permanents, 1978-1981, Linear Multilinear Algebra, 12 (1982/83), pp. 227-263.

[102] — Theory of permanents, 1982-1985, Linear Multilinear Algebra, 21 (1987), pp. 109-148.

[103] D. S. Mitrinović, R. R. Janić And J. D. KečKić, Albert Sade (1898-1973), Univ. Beograd. Publ. Elektrotehn. Fak. Ser. Mat. Fiz., 412-460 (1973), pp. 243-246.

[104] W. O. J. MoseR, The number of very reduced $4 \times n$ Latin rectangles, Canad. J. Math., 19 (1967), pp. 1011-1017.

[105] G. L. Mullen, How many $i-j$ reduced Latin squares are there?, Amer. Math. Monthly, 82 (1978), pp. 751-752.

[106] G. L. Mullen and C. Mummert, Finite Fields and Applications, American Mathematical Society, 2007.

[107] G. L. Mullen And D. PuRdy, Some data concerning the number of Latin rectangles, J. Combin. Math. Combin. Comput., 13 (1993), pp. 161-165.

[108] G. P. NAgy And P. VojtĚChOVskÝ, LOOPS - Computing with quasigroups and loops in GAP. http://www.math.du.edu/loops/

[109] _ Computing with small quasigroups and loops, Quasigroups Related Systems, 15 (2007), pp. 77-94.

[110] J. R. Nechvatal, Counting Latin Rectangles, PhD thesis, University of Southern California, 1979.

[111] — Asymptotic enumeration of generalized Latin rectangles, Util. Math., 20 (1981), pp. 273-292.

[112] D. A. Norton And S. K. Stein, An integer associated with Latin squares, Proc. Amer. Math. Soc., 7 (1956), pp. 331-334.

[113] —, Cycles in algebraic systems, Proc. Amer. Math. Soc., 7 (1956), pp. 999-1004.

[114] H. W. Norton, The $7 \times 7$ squares, Ann. Eugenics, 2 (1939), pp. 269-307.

[115] P. E. O'NeIL, Asymptotics and random matrices with row-sum and column-sum restrictions, Bull. Amer. Math. Soc., 75 (1969), pp. 1276-1282.

[116] K. T. Phelps, Latin square graphs and their automorphism groups, Ars Combin., 7 (1979), pp. 273-299.

[117] _- Automorphism-free Latin square graphs, Discrete Math., 31 (1980), pp. 193200.

[118] C. R. Pranesachar, Enumeration of Latin rectangles via SDR's, in Lecture Notes in Math., Springer, 1981, pp. 380-390.

[119] D. A. Preece, Classifying Youden rectangles, J. Roy. Statist. Soc. Ser. B, 28 (1966), pp. 118-130.

[120] J. Riondan, Three-line Latin rectangles, Amer. Math. Monthly, 51 (1944), pp. 450452. 
[121] _ A recurrence relation for three-line Latin rectangles, Amer. Math. Monthly, 59 (1952), pp. 159-162.

[122] — Book review: Percy Alexander MacMahon: Collected Papers, Volume I, Combinatorics, Bull. Amer. Math. Soc. (N.S.), 2 (1980), pp. 239-241.

[123] G.-C. Rota, On the foundations of combinatorial theory. I. Theory of Möbius functions, Z. Wahrsch. und Verw. Gebiete, 2 (1964), pp. 340-368.

[124] H. J. Ryser, Combinatorial Mathematics, Springer, 1963.

[125] A. A. SADE, Énumération des carrés latins, application au $7^{e}$ ordre, conjecture pour les ordres supérieurs, privately published, (1948). 8 pp.

[126] — Omission dans les listes de Norton pour les carrés 7 · 7, J. Reine Angew. Math., 189 (1951), pp. 190-191.

[127] — An omission in Norton's list of $7 \times 7$ squares, Ann. Math. Stat., 22 (1951), pp. 306-307.

[128] —, Quasigroupes parastrophiques. Expressions et identités, Math. Nachr., 20 (1958), pp. 73-106.

[129] —-, Quasigroupes isotopes. Autotopies d'un groupe, Annales de la Société Scientifique de Bruxelles, 81 (1967), pp. 231-239.

[130] —., Autotopies des quasigroupes et des systèmes associatifs, Arch. Math. (Brno), 4 (1968), pp. 1-23.

[131] _ Morphismes de quasigroupes. Tables, Univ. Lisboa Revista Fac. Ci. A, 13 (1970/71), pp. 149-172.

[132] P. N. Saxena, A simplified method of enumerating Latin squares by MacMahon's differential operators; I. the $6 \times 6$ squares, J. Indian Soc. Agricultural Statist., 2 (1950), pp. 161-188.

[133] — A simplified method of enumerating Latin squares by MacMahon's differential operators; II. the $7 \times 7$ squares, J. Indian Soc. Agricultural Statist., 3 (1951), pp. 2479.

[134] E. Schönhardt, Über lateinische Quadrate und Unionen, J. Reine Angew. Math., 163 (1930), pp. 183-229.

[135] A. Schrijver, A short proof of Minc's conjecture, J. Combin. Theory Ser. A, 25 (1978), pp. 80-83.

[136] E. SchröDER, Ueber v. Staudt's Rechnung mit Würfen und verwandte Processe, Math. Ann., 10 (1876), pp. 289-317.

[137] — Ueber eine eigenthümliche Bestimmung einer Function durch formale Anforderungen, J. Reine Angew. Math., 90 (1881), pp. 189-220.

[138] J.-Y. ShaO AND W.-D. WEI, A formula for the number of Latin squares, Discrete Math., 110 (1992), pp. 293-296.

[139] J. B. Shaw, On parastrophic algebras, Trans. Amer. Math. Soc., 16 (1915), pp. 361370 . 
[140] R. Sinkhorn, Concerning a conjecture of Marshall Hall, Proc. Amer. Math. Soc., 21 (1969), pp. 197-201.

[141] I. SKAU, A note on the asymptotic number of Latin rectangles, European J. Combin., 19 (1998), pp. 617-620.

[142] N. J. A. Sloane, The on-line encyclopedia of integer sequences. Published electronically at http://www.research.att.com/ njas/sequences/

[143] B. Smetaniuk, A new construction of Latin squares. II. The number of Latin squares is strictly increasing, Ars Combin., 14 (1982), pp. 131-145.

[144] J. D. H. Smith, Homotopies of central quasigroups. Submitted.

[145] R. P. Stanley, Differentiably finite power series, European J. Combin., 1 (1980), pp. $175-188$.

[146] C. M. Stein, Asymptotic evaluation of the number of Latin rectangles, J. Combin. Theory Ser. A, 25 (1978), pp. 38-49.

[147] — Approximate Computation of Expectations, Institute of Mathematical Statistics, 1986.

[148] — A way of using auxiliary randomization, in Probability Theory, Walter de Gruyter \& Co., 1992, pp. 159-180.

[149] S. K. Stein, Foundations of quasigroups, Proc. Natl. Acad. Sci. USA, 42 (1956), pp. $545-546$.

[150] —, On the foundations of quasigroups, Trans. Amer. Math. Soc., 85 (1957), pp. 228-256.

[151] D. S. Stones, The parity of the number of quasigroups. Submitted.

[152] —, (2009). http://code.google.com/p/latinrectangles/downloads/list

[153] —, On the Number of Latin Rectangles, PhD thesis, Monash University, 2010. http://arrow.monash.edu.au/hdl/1959.1/167114

[154] D. S. Stones And I. M. Wanless, Compound orthomorphisms of the cyclic group, Finite Fields Appl., 16 (2010), pp. 277-289.

[155] — A congruence connecting Latin rectangles and partial orthomorphisms. Submitted.

[156] — How not to prove the Alon-Tarsi Conjecture. Submitted.

[157] —, Divisors of the number of Latin rectangles, J. Combin. Theory Ser. A, 117 (2010), pp. 204-215.

[158] G. TARry, Le problème des 36 officers, Ass. Franç. Paris, 29 (1901), pp. 170-203.

[159] H. A. Thurston, Certain congruences on quasigroups, Proc. Amer. Math. Soc., 3 (1952), pp. 10-12.

[160] A. N. Timashov, On permanents of random doubly stochastic matrices and on asymptotic estimates for the number of Latin rectangles and Latin squares, Discrete Math. Appl., 12 (2002), pp. 431-452. 
[161] B. L. van der Waerden, Aufgabe 45, J. de Math. spéc., 35 (1926), p. 117.

[162] D. C. van Leijenhorst, Symmetric functions, Latin squares and Van der Corput's "scriptum 3", Expo. Math., 18 (2000), pp. 343-356.

[163] J. H. van Lint and R. M. Wilson, A Course in Combinatorics, Cambridge University Press, 1992.

[164] I. M. WANLESS, Perfect factorisations of bipartite graphs and Latin squares without proper subrectangles, Electron. J. Combin., 6 (1999). R9, 16 pp.

[165] _ - A lower bound on the maximum permanent in $\Lambda_{n}^{k}$, Linear Algebra Appl., 373 (2003), pp. 153-167.

[166] _ - Atomic Latin squares based on cyclotomic orthomorphisms, Electron. J. Combin., 12 (2005). R22, 23 pp.

[167] I. M. WANless AND E. C. Ihrig, Symmetries that Latin squares inherit from 1-factorizations, J. Combin. Des., 13 (2005), pp. 157-172.

[168] M. B. WeLLs, The number of Latin squares of order eight, J. Combin. Theory, 3 (1967), pp. 98-99.

[169] — Elements of Combinatorial Computing, Pergamon Press, 1971.

[170] H. Whitney, A logical expansion in mathematics, Bull. Amer. Math. Soc., 38 (1932), pp. 572-579.

[171] H. S. WiLf, What is an answer?, Amer. Math. Monthly, 89 (1982), pp. 289-292.

[172] K. Yамамото, On the asymptotic number of Latin rectangles, Japan. J. Math., 21 (1951), pp. 113-119.

[173] _ Note on enumeration of $7 \times 7$ Latin squares, Bull. Math. Statist., 5 (1952), pp. 1-8.

[174] —_, On Latin squares, Sûgaku, 12 (1960/61), pp. 67-79. In Japanese.

[175] _ On the number of Latin rectangles, Res. Rep. Sci. Div. Tokyo Womens' Univ., 19 (1969), pp. 86-97.

[176] P. ZAPPA, The Cayley determinant of the determinant tensor and the Alon-Tarsi Conjecture, Adv. in Appl. Math., 13 (1997), pp. 31-44.

[177] D. Zeilberger, Enumerative and algebraic combinatorics, in Princeton Companion to Mathematics, Princeton University Press, 2008, pp. 550-561.

[178] C. Zhang And J. Ma, Counting solutions for the $N$-queens and Latin square problems by Monte Carlo simulations, Phys. Rev. E, 79 (2009). 016703. 